\title{
MINIMUM SIMPLICIAL COMPLEXES WITH GIVEN ABELIAN AUTOMORPHISM GROUP ${ }^{1}$
}

BY

\author{
ZEVI MILLER
}

\begin{abstract}
Let $K$ be a pure $n$-dimensional simplicial complex. Let $\Gamma_{0}(K)$ be the automorphism group of $K$, and let $\Gamma_{n}(K)$ be the group of permutations on $n$-cells of $K$ induced by the elements of $\Gamma_{0}(K)$. Given an abelian group $A$ we consider the problem of finding the minimum number of points $M_{0}^{(n)}(A)$ in $K$ such that $\Gamma_{0}(K) \cong A$, and the minimum number of $n$-cells $M_{1}^{(n)}(A)$ in $K$ such that $\Gamma_{n}(K) \cong A$. Write $A=\prod_{p^{\alpha}} \mathbf{Z}_{p^{\alpha}}^{e\left(p^{\alpha}\right)}$, where each factor $\mathbf{Z}_{p^{\alpha}}$ appears $e\left(p^{\alpha}\right)$ times in the canonical factorization of $A$. For $A$ containing no factors $\mathbf{Z}_{p^{\alpha}}$ satisfying $p^{\alpha}<17$ we find that $M_{1}^{(n)}(A)=M_{0}^{(2)}(A)=\Sigma_{p^{\alpha}} p^{\alpha} e\left(p^{\alpha}\right)$ when $n \geqslant 4$, and we derive upper bounds for $M_{1}^{(n)}(A)$ and $M_{0}^{(n)}(A)$ in the remaining possibilities for $A$ and $n$.
\end{abstract}

1. Introduction. A simplicial complex $K$ is defined as a pair $(V, \delta)$ where $V$ is a finite set (whose elements are called points) and $\delta$ is a set of subsets of $V$ (called simplexes or cells) with the property that $\beta \in \mathcal{S}$ and $\varnothing \neq \alpha \subset \beta$ imply $\alpha \in \mathcal{S}$, while $x \in V$ implies $\{x\} \in \mathcal{S}$. The dimension of a simplex $\beta$ having $n+1$ points is $n$, and the dimension of a complex $K$ is the maximum dimension of any simplex of $K$. We also say that $K$ is a pure $r$-dimensional simplicial complex (or an $r$-complex) if $K$ has dimension $r$ and the maximal simplexes of $K$ all have dimension $r$.

We will be concerned with pure $n$-dimensional simplicial complexes for $n \geqslant 2$. When $n=2$, such a complex will be called a plex for brevity. Following the notation of Dewdney [3] we denote by $K_{(r)}$ the set of $r$-cells of $K$, so that in particular if we identify each point $x$ with the 0 -simplex $\{x\}$ we get $K_{(0)}=V$. If $S \subset V$ then we define $\langle S\rangle$, the complex induced by $S$, as the complex having $S$ as point set and having as simplexes those subsets of $S$ which are simplexes in $K$. If $D \subset \delta$, define $\langle D\rangle$, the complex induced by $D$, as follows. We define the point set $V(\langle D\rangle)$ by $V(\langle D\rangle)=\cup_{\alpha \in D}\{x \in \alpha: x \in V\}$ and the set of simplexes $\delta(\langle D\rangle)$ by $\delta(\langle D\rangle)=$ $\cup_{\alpha \in D}\{\beta \in \mathcal{S}: \beta \subset \alpha\}$. If $v \in V(K)$ and $K$ has dimension $n$, then the set points in $V-v$ which are contained in $n$-cells with $v$ will be called the neighborhood of $v$ and will be denoted by $N(v)$. A 1-1 and onto map $f: V(K) \rightarrow V\left(K^{\prime}\right)$ between $n$-dimensional complexes $K$ and $K^{\prime}$ is called simplicial when $\alpha \in K_{(n)}$ if and only if $\langle\{f(x)$ : $x \in \alpha\}\rangle \in K_{(n)}^{\prime}$. If such a map $f$ exists, we say $K$ and $K^{\prime}$ are isomorphic and we write $K \cong K^{\prime}$. If $L$ is an induced subcomplex of $K$, we write $f(L)$ for $\langle\{f(l): l \in V(L)\}\rangle$.

Received by the editors February 22, 1980 and, in revised form, April 7, 1981.

1980 Mathematics Subject Classification. Primary 05C65; Secondary 05C25.

'This paper is based on the author's doctoral dissertation in the Department of Mathematics at the University of Michigan. The author was partially supported by a Miami University summer research award during the preparation of this paper. 
The point group and cell group of an $n$-complex are defined in the natural way. We let $\Gamma_{0}(K)$, the point group of $K$, be the set of all functions $f: V \rightarrow V$ which are simplicial. The subgroup of $\Gamma_{0}(K)$ consisting of all elements fixing $v \in V(K)$ will be denoted by $\Gamma_{0}(K)_{v}$. When the complex $K$ is understood, $\Gamma_{0}(K)_{v}$ may be abbreviated $\left(\Gamma_{0}\right)_{v}$. An automorphism $\sigma$ in $\Gamma_{0}(K)$ naturally induces a permutation $\tilde{\sigma}$ on $K_{(n)}$ as follows. If $\alpha, \beta \in K_{(n)}$, then we write $\tilde{\sigma}(\alpha)=\beta$ if $\sigma\langle\alpha\rangle=\langle\beta\rangle$. The set of permutations on $K_{(n)}$ given by $\left\{\tilde{\sigma}: \sigma \in \Gamma_{0}(K)\right\}$ is clearly a group, will be called the cell group of $K$, and will be denoted by $\Gamma_{n}(K)$. Hence there is a surjective homomorphism $\pi$ : $\Gamma_{0}(K) \rightarrow \Gamma_{n}(K)$ defined by $\pi(\alpha)=\tilde{\alpha}$. Finally when $\sigma$ is a group element, $\langle\sigma\rangle$ will denote the subgroup generated by $\sigma$, and $\{x\}$ will denote the least integer greater than or equal to the real number $x$.

The purpose of this investigation is to study the following problem. Given an arbitrary finite abelian group $A$, find the minimum number of $n$-cells $M_{1}^{(n)}(A)$ in an $n$-complex $K$ satisfying $\Gamma_{n}(K)=A$, and find the minimum number of points $M_{0}^{(n)}(A)$ in an $n$-complex $K$ satisfying $\Gamma_{0}(K)=A$.

Let $A=\prod \mathbf{Z}_{p^{\alpha}}^{e\left(p^{\alpha}\right)}$ via the decomposition theorem for abelian groups, where $e\left(p^{\alpha}\right)$ is the number of direct factors isomorphic to $\mathbf{Z}_{p^{\alpha}}$ for a fixed $p^{\alpha}$. We solve the problem of determining $M_{1}^{(n)}(A)$ when $n \geqslant 4$ for those $A$ all of whose direct factors $\mathbf{Z}_{p^{\alpha}}$ satisfy $p^{\alpha} \geqslant 17$. The same problem is solved when $n=2$ or 3 for those $A$ having the additional property of $e\left(p^{\alpha}\right)$ being bounded by a certain function of $p^{\alpha}$ for all $p^{\alpha}$. We also determine $M_{0}^{(2)}(A)$ for those $A$ all of whose direct factors $\mathbf{Z}_{p^{\alpha}}$ satisfy $p^{\alpha} \geqslant 9$.

Given the group $A$ and the integer $n$, we construct $n$-dimensional simplicial complexes $K_{1}^{(n)}(A)$ and $K_{0}^{(n)}(A)$ having cell automorphism group and automorphism group (respectively) isomorphic to $A$, that is, $\Gamma_{n}\left(K_{1}^{(n)}(A)\right) \cong A$ and $\Gamma_{0}\left(K_{0}^{(n)}(A)\right) \cong A$. These constructions form the crux of this work, and are used in Theorems 8,9 , and 10 to give upper bounds for $M_{1}^{(n)}(A)$ and $M_{0}^{(n)}(A)$ for abelian groups $A$ and integers $n$. The required lower bounds are found by determining (in Lemma 11.1) the minimum degree in any faithful action of an arbitrary abelian group. These facts are combined to yield our main result, Theorem 11, which gives the exact values of $M_{1}^{(n)}(A)$ and $M_{0}^{(2)}(A)$.

The motivation for this work originates with results of Arlinghaus [1], Meriwether [10], Babai [2], and Sabidussi [12] for graphs, i.e., dimension 1. Meriwether first found the minimum number of points in a graph with given finite cyclic automorphism group, and Arlinghaus generalized this result to cover arbitrary finite abelian groups.

As $n$ varies it is interesting to observe the relationship among the exact values and bounds for $M_{1}^{(n)}(A)$ and $M_{0}^{(n)}(A)$, and among the constructions used in realizing these bounds. It will be seen that as $n$ increases these numbers and the constructions from which they arise assume a rather simple and elegant form. This would present an example of the interesting phenomenon in which one is able to better understand a geometric invariant as the dimension of the space in question increases. In this regard, we note that the determination of $M_{1}^{(1)}(A)$, the minimum number of lines in a graph with line group $A$, is completely open and is an extremely difficult problem. 
It was also found that the class of 2-complexes which realize the numbers $M_{1}^{(2)}\left(\mathbf{Z}_{p^{\alpha}}\right)$ and $M_{0}^{(2)}\left(Z_{p^{\alpha}}\right)$ was unique and could be utilized, by means of the topological cone, to give the complexes which yielded the values of $M_{1}^{(n)}(A)$ and $M_{0}^{(n)}(A)$, if $n \geqslant 2$, for all finite abelian groups $A$. It is precisely the fact that $A$ is a direct product of prime power cyclic subgroups which makes this construction possible. In the same way, it is expected that a determination of $M_{1}^{(n)}(G)$ and $M_{0}^{(n)}(G)$ for $p$-groups $G$ should yield upper bounds (which are probably exact) for $M_{1}^{(n)}(H)$ and $M_{0}^{(n)}(H)$ when $H$ is nilpotent.

The text is organized as follows. In $\S 2$ a set $C$ of 2-complexes is defined, each member $K$ of which satisfies $\left|K_{(2)}\right|=p^{\alpha}$ and $\Gamma_{2}(K) \supset \mathbf{Z}_{p^{\alpha}}$ for given $p$ and $\alpha$. A characterization is then found for the subset $C^{\prime} \subset C$ consisting of complexes $K^{\prime}$ satisfying $\Gamma_{2}\left(K^{\prime}\right) \not \mathbf{Z}_{p^{\alpha}}$, and a formula is developed for the number of isomorphism classes among the set of 2-complexes in $C \backslash C^{\prime}$. From the resulting fact that $C \backslash C^{\prime}$ is nonempty for $p^{\alpha} \geqslant 9$, it follows that $M_{1}^{(2)}\left(\mathbf{Z}_{p^{\alpha}}\right)=p^{\alpha}$ for $p^{\alpha} \geqslant 9$. We conclude $\S 2$ with a characterization of all 2-complexes $K$ satisfying $\left|K_{(2)}\right|=p^{\alpha}$ and admitting an element in $\Gamma_{2}(K)$ which is a $p^{\alpha}$-cycle. One consequence of this result is that the set of 2-complexes $K$ satisfying $\left|K_{(2)}\right|=p^{\alpha}$ and $\Gamma_{2}(K) \cong \mathbf{Z}_{p^{\alpha}}$ is shown to be identical with $C \backslash C^{\prime}$. In $\S 3$ bounds for $M_{1}^{(n)}(A)$ and $M_{0}^{(n)}(A)$ are obtained by construction. Letting $A=\prod \mathbf{Z}_{p^{\alpha}}^{e\left(p^{\alpha}\right)}$, we construct the $n$-complexes $K_{1}^{(n)}(A)$ and $K_{0}^{(n)}(A)$, which yield the upper bounds for $M_{1}^{(n)}(A)$ and $M_{0}^{(n)}(A)$ respectively, by forming them, in all but certain "small" cases, as disjoint unions of the complexes $K_{i}^{(n)}\left(\mathbf{Z}_{p^{\alpha}}^{e\left(p^{\alpha}\right)}\right), i=0,1$, over distinct prime powers appearing in the factorization of $A$. The characterization and enumeration in $\$ 2$ is used in determining which prime powers $p^{\alpha}$ admit the construction $K_{i}^{(n)}\left(\mathbf{Z}_{p^{\alpha}}^{e\left(p^{\alpha}\right)}\right), i=0,1$, in effect, how large $p^{\alpha}$ must be.

A general description of the method used in constructing $K_{1}^{(n)}\left(\mathbf{Z}_{p^{\alpha}}^{e\left(p^{\alpha}\right)}\right)$ is in order here. We begin by choosing a pair, say $R$ and $S$, of nonisomorphic 2-complexes from the set $C \backslash C^{\prime}$. By the result of $\S 2$, each of these is known to have cell group $\mathbf{Z}_{p^{\alpha}}$. We then attach $e\left(p^{\alpha}\right)-1$ copies of $R$ and one copy of $S$ together to form a kind of "tower" complex having $e\left(p^{\alpha}\right)$ "floors". The bottom floor of this tower is a cone over $R$, while each of the top $e\left(p^{\alpha}\right)-1$ floors is isomorphic to a cone over $S$. From the nature of the coning operation (to be defined in \$3) it will follow that the cell group of each floor is the same as the cell group of either $R$ or $S$. We will then see that the cell group of the entire tower contains the direct product $\mathbf{Z}_{p^{\alpha}}^{e\left(p^{\alpha}\right)}$, this group arising from the action of $\mathbf{Z}_{p^{\alpha}}$ on each of the $e\left(p^{\alpha}\right)$ floors independently. That the cell group of the tower contains nothing more than $\mathbf{Z}_{p^{\alpha}}^{e\left(p^{\alpha}\right)}$, i.e., that

$$
\Gamma_{n}\left(K_{1}^{(n)}\left(\mathbf{Z}_{p^{\alpha}}^{e\left(p^{\alpha}\right)}\right)\right)=\mathbf{Z}_{p^{\alpha}}
$$

will follow from the nature of the tower construction. Specifically, any element of the cell group lying outside of the subgroup $\mathbf{Z}_{p^{\alpha}}^{e\left(p^{\alpha}\right)}$ just described must be a nonidentity permutation on the floors of the tower (since all elements acting invariantly on each floor are accounted for in the subgroup $\left.\mathbf{Z}_{p^{\alpha}}^{e\left(p^{\alpha}\right)}\right)$. But such a permutation will be impossible since the tower has been "rooted" at its bottom floor. That is, a nonidentity permutation of the floors must move the bottom floor to 
some other floor (by the nature of the construction), and this is impossible since $R$ and $S$ were chosen to be nonisomorphic complexes.

The complexes $K_{0}^{(n)}\left(\mathbf{Z}_{p^{\alpha}}^{e\left(p^{\alpha}\right)}\right)$ are formed using an essentially different method, based on a higher-dimensional version of graph complementation. In these complexes too, however, the basic building blocks are the 2-dimensional complexes in the set $C \backslash C^{\prime}$.

Finally, we remark that our main result, Theorem 11, gives $M_{0}^{(2)}(A)=M_{1}^{(n)}(A)=$ $\sum p^{\alpha} e\left(p^{\alpha}\right), n \geqslant 4$, for all $A$ with the exception of certain "small" cases.

2. A set of extremal complexes. Throughout this section the word "cell" will be used interchangeably with 2-cell. Our first task will be to construct plexes having $p^{\alpha}$ cells and cyclic cell group $\mathbf{Z}_{p^{\alpha}}$. It will be seen that this is possible for $p^{\alpha} \geqslant 9$. These plexes will later be used in the construction of $r$-complexes, $r \geqslant 2$, that provide upper bounds for $M_{i}^{(r)}(A), i=0,1$, and $A$ finite abelian.

Let $n=p^{\alpha}$ be a fixed prime power. We will define a set $C$ of plexes, each having $n$ 2-cells. For $j$ satisfying $3 \leqslant j \leqslant n$, form the plex $C(n, j)$ by letting $V(C(n, j))=$ $\{1,2,3, \ldots, n\}$, and $C(n, j)_{(2)}=\{\langle 1+i, 2+i, j+i\rangle: 0 \leqslant i \leqslant n-1\}$, addition performed modulo $n$. We illustrate the 1-skeleton of the complex $C(8,4)$ in Figure 2.0.

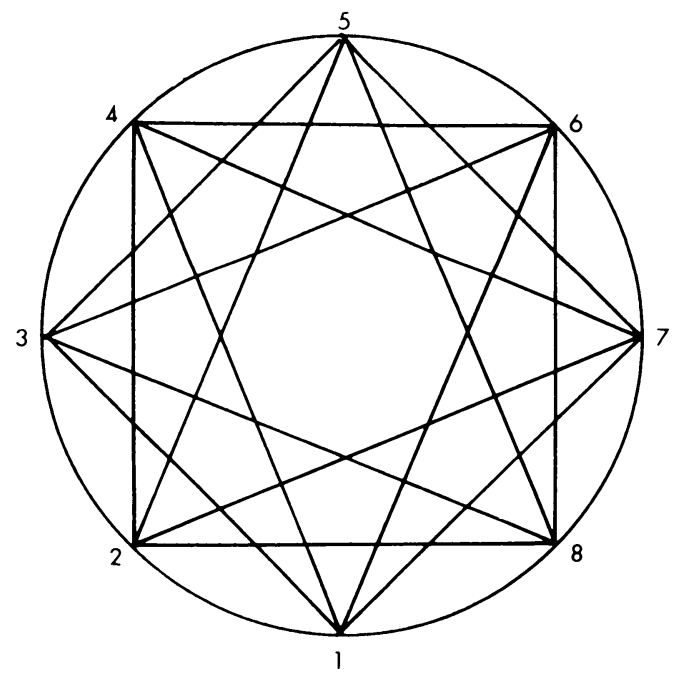

FIGURE 2.0. The 1-skeleton of $C(8,4)$

Observe that there exists an isomorphism $f: C(n, j) \rightarrow C(n, n+3-j)$ given by $f(k)=n+3-k, 1 \leqslant k \leqslant n$. Thus for $n$ even there is a one-to-one correspondence between the two sets of complexes $S=\{C(n, j): 3 \leqslant j \leqslant\{n / 2\}+1\}$ and $T=$ $\{C(n, j):\{n / 2\}+2 \leqslant j \leqslant n\}$ given by $C(n, j) \leftrightarrow C(n, n+3-j), 3 \leqslant j \leqslant\{n / 2\}$ +1 , where corresponding complexes are isomorphic. For $n$ odd the two sets are $S=\{C(n, j): 3 \leqslant j \leqslant\{n / 2\}\}$ and $T=\{C(n, j):\{n / 2\}+2 \leqslant j \leqslant n\}$, the complex $C(n,\{n / 2\}+1)$ corresponding to itself under $f$. Hence, for all $n$, the set $C$ of 
complexes defined by $C=\{C(n, j): 3 \leqslant j \leqslant\{n / 2\}+1\}$ contains at least one complex from each isomorphism class in the collection of complexes $C(n, j)$, $3 \leqslant j \leqslant n$. In analyzing the complexes $C(n, j)$, we therefore restrict our attention to the subset $C$ of them. Notice that for each member $C(n, j)$ of $C$ there exists an element $\tau=(12 \cdots n)$ of $\Gamma_{0}(C(n, j))$ and that $\langle\tau\rangle \cong \mathbf{Z}_{n}$. We also have $\langle\tilde{\tau}\rangle \cong \mathbf{Z}_{n}$ as a subgroup of $\Gamma_{2}(C(n, j))$, and it follows that $\Gamma_{2}(C(n, j)) \supset \mathbf{Z}_{n}$ for all $C(n, j) \in C$.

We begin by isolating those plexes $C(n, j)$ of $C$ which satisfy $\Gamma_{2}(C(n, j)) \cong \mathbf{Z}_{n}$. Let $C^{\prime}$ be the subset of $C$ consisting of plexes $C(n, j)$ whose parameters $n$ and $j$ satisfy at least one of the following conditions:

$1 . n \leqslant 8$,

2. $j=3$,

3. $n=2 j, n>16$,

4. $n=2 j-2, n \geqslant 16$,

5. $n=2 j-3, n \geqslant 9$,

6. $j^{2}-3 j+3 \equiv 0(\bmod n), p \equiv 1(\bmod 6)$.

In Theorem 1 we show that no member $C(n, j)$ of $C^{\prime}$ satisfies $\Gamma_{2}(C(n, j)) \cong \mathbf{Z}_{n}$. In the considerably more involved Theorem 2 it is proved that $\Gamma_{2}(C(n, j)) \cong \mathbf{Z}_{n}$ if and only if $C(n, j) \in C \backslash C^{\prime}$.

Theorem 1. Let $C(n, j) \in C^{\prime}$, then we have $\Gamma_{2}(C(n, j)) \not \mathbf{Z}_{n}$.

Proof. As noted previously, we already know that $\Gamma_{2}(C(n, j)) \supset \mathbf{Z}_{n}=\langle\tilde{\tau}\rangle$ for any $n$ and $j$. Hence for each $C(n, j) \in C^{\prime}$ we need only construct an element $\sigma \in \Gamma_{0}(C(n, j))$ such that $\tilde{\sigma} \notin\langle\tilde{\tau}\rangle$.

Suppose first that $j=3$ in $C(n, j)$. It has already been observed that $\sigma=$ $\Pi\left\{_{k=1}^{n / 2\}+1}(k, n+3-k)\right.$ gives an isomorphism $\sigma: C(n, 3) \rightarrow C(n, n)$. Since $C(n, n)$ is identical with $C(n, 3)$, it follows that $\sigma$ may be viewed as an element of $\Gamma_{0}(C(n, 3))$. Clearly $\tilde{\boldsymbol{\sigma}} \notin\langle\tilde{\tau}\rangle$, and hence $\Gamma_{0}(C(n, 3)) \not \mathbf{Z}_{n}$, as required.

Now suppose $n=2 j-3$. We will show that $C(n, j) \cong C(n, 3)$. To do this, define the map $f: C(n, 3) \rightarrow C(n, j)$ given by $f(k)=1+(k-1)\{n / 2\}$. First we show that $f$ is 1-1. For if not, then for two distinct points $k_{1}, k_{2} \in C(n, 3)$ we would have $f\left(k_{1}\right) \equiv f\left(k_{2}\right)(\bmod n)$, that is, $1+\left(k_{1}-1\right)\{n / 2\} \equiv 1+\left(k_{2}-1\right)\{n / 2\}(\bmod n)$. Since $n$ is an odd prime power we have $(\{n / 2\}, n)=1$ and hence $k_{1} \equiv k_{2}(\bmod n)$. This shows that $f$ is one-one, and since it is a map between finite sets it must also be onto. It remains to show that $f$ is simplicial. By the linearity in the definition of $f$, it suffices to show that $f\langle 1,2,3\rangle \in C(n, j)_{(2)}$. But this follows immediately on observing that $f(1)=1, f(2)=1+\{n / 2\}=j$, and $f(3)=1+2\{n / 2\}=n+2 \equiv 2$. Thus the assertion $C(n, j) \cong C(n, 3)$ is proved. It follows that $\Gamma_{2}(C(n, j)) \cong \Gamma_{2}(C(n, 3))$ $\not \mathbf{Z}_{n}$.

Let us now pass to the case $n=2 j-2$. Here we define $\sigma$ as

$$
\boldsymbol{\sigma}=(1)(j) \prod_{k=2}^{j-1}(k, j+k-1) .
$$


It is obvious that $\sigma$ is one-one and onto. To see that $\sigma$ is simplicial, take a typical 2-cell $D=\langle k, k+1, k+j-1\rangle$. By definition $\sigma(D)=\langle j+k-1, j+k, k\rangle$, and noting that $2 j+k-2 \equiv k(\bmod n)$ we get $\sigma(D)=\langle j+k-1, j+k, 2 j+k-$ $2\rangle \in C(n, j)_{(2)}$, as required. Clearly $\tilde{\sigma} \notin\langle\tilde{\tau}\rangle$ since $\sigma$ has two fixed points.

Now suppose $n=2 j, n \geqslant 16$. We define $\sigma$ by

$$
\sigma=(n)(n / 2) \prod_{\substack{k \text { odd } \\ n / 4<k<3 n / 4}}(k, 3 n / 2-k) \prod_{\substack{k \text { even } \\ k<n / 2}}(k, n-k) .
$$

It is perhaps easiest to understand $\sigma$ by considering the following "intuitive" description of it. We let $\sigma$ act on the even numbered points as a reflection about the pointwise fixed axis $\{n, n / 2\}$, and on the odd numbered points as a reflection about the axis $\{n / 4,3 n / 4\}$ while interchanging the two points of this last axis. Clearly then $\sigma$ is $1-1$ and onto. Let us now show that $\sigma$ is simplicial by considering its action on a typical 2-cell $D=\langle k, k+1, k+(n / 2)-1\rangle$. By symmetry and from the description of $\sigma$ given above, we may assume without loss of generality that $k$ satisfies $n / 4 \leqslant k \leqslant n / 2$. Suppose first $k$ is odd. Then observing that $3 n / 2 \equiv n / 2$ $(\bmod n)$ and writing $k+(n / 2)-1$ as $n-((n / 2)-k+1)$ we find that $\sigma(D)=$ $\langle(n / 2)-k,(n / 2)-k+1,(n / 2)-k+(n / 2)-1\rangle \in C(n, j)_{(2)}$, as required. Now suppose $k$ is even. Here we rewrite $k+(n / 2)-1$ as $3(n / 2)-(n-k+1)$ and we get $\sigma(D)=\langle n-k, n-k+1, n-k+((n / 2)-1)\rangle \in C(n, j)_{(2)}$. Thus $\sigma$ is simplicial and hence it is an automorphism of $C(n, j)$. Finally, we note that $\tilde{\sigma} \notin\langle\tilde{\tau}\rangle$ since $\sigma$ has two fixed points.

Now suppose $j^{2}-3 j+3 \equiv 0(\bmod n)$ where $n$ is a power of a prime $p$ satisfying $p \equiv 1(\bmod 6)$. We define $\sigma$ by $\sigma(r)=3 j-3+r(1-j)$ for $r \in C(n, j)$. Let $D=\langle r, r+1, r+j-1\rangle$ be a typical 2-cell of $C(n, j)$. We get by definition $\sigma\langle r, r+1, r+j-1\rangle=\left\langle z_{1}, z_{2}, z_{3}\right\rangle$, where $z_{1}=3 j-3+r(1-j), z_{2}=3 j-3+$ $(r+j)(1-j), z_{3}=3 j-3+(r+j-1)(1-j)$. Observe that $z_{1}-z_{2}=j-1$, so that to show $\sigma$ is simplicial we need only show that $z_{3} \equiv z_{2}+1(\bmod n)$. This in turn is equivalent to $(r+1-1)(1-j) \equiv 1+(r+1)(1-j)(\bmod n)$. But we have, using simple manipulation and $j^{2}-3 j+3 \equiv 0(\bmod n)$, that $(r+j-1)(1-j)=$ $(r+1)(1-j)-j^{2}+3 j-2 \equiv(r+1)(1-j)+1(\bmod n)$, as desired. We remark that the condition $p \equiv 1(\bmod 6)$ arises since the congruence $j^{2}-3 j+3 \equiv 0$ $\left(\bmod p^{\alpha}\right)$ has solutions (exactly two in fact) if and only if $p \equiv 1(\bmod 6)$. To see this, first reduce to the equivalent congruence $(2 j-3)^{2} \equiv-3\left(\bmod p^{\alpha}\right)$. It is well known $\left[13\right.$, p. 94] that if $p=2$ and $\alpha \geqslant 4$, then -3 is not a quadratic residue $\bmod p^{\alpha}$. Hence we may restrict our attention to odd primes. By employing formal Taylor series one may prove that $(2 j-3)^{2} \equiv-3\left(\bmod p^{\alpha}\right)$ has either 2 or no solutions according to whether -3 is a quadratic residue mod $p$ or not. We are thus reduced to determining the Legendre symbol $\left(\frac{-3}{p}\right)$. By elementary properties of the symbol and quadratic reciprocity we have

$$
\left(\frac{-3}{p}\right)=\left(\frac{-1}{p}\right)\left(\frac{3}{p}\right)=\left(\frac{-1}{p}\right)\left(\frac{p}{3}\right)(-1)^{(p-1) / 2}=\left(\frac{p}{3}\right) .
$$


Hence there is a solution if and only if $p \equiv 1(\bmod 3)$, which combined with $p$ being odd implies that $p \equiv 1(\bmod 6)$, as required.

Finally, if $n \leqslant 8$ one may verify directly that there exist nontrivial elements of $\Gamma_{0}(C(n, j))$ having fixed points, and hence in these cases too one has $\Gamma_{2}(C(n, j)) \not$ $\mathbf{Z}_{n}$. When $n=7$, we know in fact that $C(7, j)$ for any $j$ is isomorphic to the projective plane on seven points and hence $\left|\Gamma_{0}(C(n, j))\right|=168$.

In order to prove the converse to Theorem 1, we will require additional terminology. Suppose $f: C(n, j) \rightarrow C(n, k)$ is an isomorphism of plexes with the property $f\langle 1,2, j\rangle=\langle 1,2, k\rangle$. The inverse image $f^{-1}\langle 1,2\rangle$ can be any one of three possible 1 -cells, and each of these may be mapped onto $\langle 1,2\rangle$ in two ways. This suggests six classes of isomorphisms satisfying $f\langle 1,2, j\rangle=\langle 1,2, k\rangle, T^{*}(1,2), T^{*}(2,1), T^{*}(1, j)$, $T^{*}(j, 1), \quad T^{*}(2, j), \quad T^{*}(j, 2)$ where we define $T^{*}(a, b)$ by $T^{*}(a, b)=\{f$ : $C(n, j) \stackrel{\simeq}{\rightarrow} C(n, k): f\langle 1,2, j\rangle=\langle 1,2, k\rangle, f(a)=1, f(b)=2\}$. We remark that if a pair of complexes $C(n, j)$ and $C(n, k)$ are isomorphic, then the set of maps $f$ as defined above, denoted by $T^{*}(n, j, k)$, is the disjoint union of the six classes given. The indices $j, k$, and $n$ are omitted from the symbols $T^{*}(a, b)$ as they will be understood by context. Suppose that $f \in T^{*}(a, b),(|b-a|, n)=1$, and that $f$ has the additional property $f(a+(r-1)(b-a))=r, 1 \leqslant r \leqslant n$, with all integers read modulo $n$. Thus $f$ maps the hamiltonian cycle $a, b, 2 b-a, 3 b-2 a, \ldots, a$ in $C(n, j)$ onto the hamiltonian cycle $1,2, \ldots, n$ in the image $C(n, k)$. This map $f$ will be denoted by $T(a, b)$, and thus $T(a, b) \in T^{*}(a, b)$.

Let us make some observations concerning the maps $T(a, b)$ acting on a fixed plex $C(n, j)$ as domain. From the definition it is clear that $T(a, b)^{-1}$ is given by $T(a, b)^{-1}(x)=a+(x-1)(b-a)$ and hence $T(a, b)(x)=1+(x-a) /(b-a)$. Therefore given the ordered pair $(a, b)$, the image complex $T(a, b)[C(n, j)]$ is uniquely determined. For example, if we write $T(1, j)[C(n, j)]=C(n, k)$, then $T(1, j)(2)=k$ and thus $k$ is determined by $k=T(1, j)(2)=(j-1)^{-1}+1$, inverses being taken in the group of units in $\mathbf{Z}_{n}$. The fact that $T(1, j): C(n, j) \rightarrow$ $C\left(n,(j-1)^{-1}+1\right)$ is an isomorphism can be verified by considering its action on an arbitrary 2-cell $\langle x, x+1, x+j-1\rangle$ of $C(n, j)$. Letting

$$
z=1+(x-1) /(j-1)
$$

and using $k=(j-1)^{-1}+1$ we find that $T(1, j)\langle x, x+1, x+j-1\rangle=\langle z, z+k$ $-1, z+1\rangle \in C(n, k)_{(2)}$. Furthermore, $T(1, j)$ is $1-1$ since $(j-1, n)=1$, and hence it is onto by finiteness of $n$. Now given any of the five remaining ordered pairs $(a, b)$ one may in a similar way find the uniquely determined $k$ for which $T(a, b)[C(n, j)]=C(n, k)$, and hence prove that $T(a, b)$ is an isomorphism. The results are summarized in Table 1 for later reference. Observe that the pairs of plexes $T(a, b)[C(n, j)]=C(n, k)$ and $T(b, a)[C(n, j)]=C(n, n+3-k)$ are related by a reflection about the axis $\{1, n / 2+1\}$ when $n$ is even and the axis formed by $\{n / 2\}+1$ and the "midpoint" of the 1 -cell $\langle 1, n\rangle$ when $n$ is odd. 
TABLE 1. The values of $k$ for which $T(a, b)[C(n, j)]=C(n, k)$

\begin{tabular}{l|l}
$(a, b)$ & $k$ \\
\hline$(1,2)$ & $j$ \\
\hline$(2,1)$ & $n+3-j$ \\
\hline$(1, j)$ & $(j-1)^{-1}+1$ \\
\hline$(j, 1)$ & $n+3-(j-1)^{-1}-1$ \\
\hline$(2, j)$ & $-(j-2)^{-1}+1$ \\
\hline$(j, 2)$ & $n+3+(j-2)^{-1}-1$ \\
\hline
\end{tabular}

For a fixed $j$, we will denote by $T^{j}$ the set of isomorphisms $\{T(a, b):\{a, b\} \subset$ $\{1,2, j\}\}$ defined above. The elements of $T^{j}$ will often be referred to as division isomorphisms.

Let $f: C(n, j) \rightarrow C(n, k)$ be an isomorphism between two complexes in $C \backslash C^{\prime}$. By a translation of the indices in $V(C(n, k))$ we may suppose that $f \in T^{*}(a, b)$ for some ordered pair $(a, b)$. Our first objective is to show that in fact $f=T(a, b)$. That is, all isomorphisms (up to a translation) are division isomorphisms. We will require the following observation, stated as a lemma for future reference.

Lemma 2.1. Let $C(n, k) \in C \backslash C^{\prime}$. Then $\langle k-1, k, 1\rangle$ is not a 2-cell of $C(n, k)$.

Proof. If not, then we must have $n+1=2 k-2$ or $2 k-3$, both contradicting $C(n, k) \in C \backslash C^{\prime}$.

We are now ready for the theorem which will lead to the converse of Theorem 1 . In the proof and subsequent discussion, $C(n, j)$ will often be abbreviated as $C(j)$ since $n$ will be fixed by context.

THEOREM 2. Suppose $n \geqslant 9$ and that two complexes $C(n, j), C(n, k) \in C \backslash C^{\prime}$ are isomorphic, say, by the isomorphism $f: C(n, j) \rightarrow C(n, k)$. Then $f$ is a division isomorphism, up to a translation of the indices of $C(n, k)$. That is, $f=\tau^{r} \circ T(a, b)$ for some $r$ and some ordered pair $(a, b)$, where $\tau=(123 \cdots n) \in \Gamma_{0}(C(n, k))$.

Proof. First some preliminary remarks are in order. As a convention, the points of $C(n, j)$ will be unprimed while those of $C(n, k)$ will be primed. Also it should be emphasized that the hypothesis requires both $C(n, j)$ and $C(n, k)$ to be in $C \backslash C^{\prime}$, so that in particular we have $4 \leqslant j, k \leqslant\{n / 2\}$. Thus, for example, if $\left\langle r^{\prime}, r+2^{\prime}\right\rangle \in$ $C(k)_{(1)}$ for some $r^{\prime} \in V(C(k))$ we may conclude that $k=4$, a conclusion which would be false without the above restriction on $k$.

Our method is as follows. We prove that for each ordered pair $(a, b)$, if $g$ is an isomorphism in $T^{*}(a, b), g: C(n, j) \rightarrow C(n, k)$, then $g=T(a, b)$. Note that this suffices to prove the theorem. For let $f$ be any isomorphism, $f: C(n, j) \rightarrow C(n, k)$, and suppose that $f\langle 1,2, j\rangle=\left\langle r^{\prime}, r+1^{\prime}, r+k-1^{\prime}\right\rangle$. Then letting $\tau_{2}=\left(1^{\prime} 2^{\prime} 3^{\prime}\right.$ $\left.\cdots n^{\prime}\right) \in \Gamma_{0}(C(k))$, we have $\tau_{2}^{-(r-1)} f\langle 1,2, j\rangle=\left\langle 1^{\prime}, 2^{\prime}, k^{\prime}\right\rangle$, and hence $\tau_{2}^{-(r-1)} f \in$ $T^{*}\left(a_{0}, b_{0}\right)$ for some ordered pair $\left(a_{0}, b_{0}\right)$. Now letting $g=\tau_{2}^{-(r-1)} f$, we know by the 
result above that $\tau_{2}^{-(r-1)} f=T(a, b)$, and the theorem will be proved. Observe further that to show $g=T(a, b)$ it suffices to prove that $g(2 b-a)=3^{\prime}$. For then letting $\tau_{1}=(12 \cdots n) \in \Gamma_{0}(C(j))$ we see that the map

$$
\tau_{2}^{-1} g \tau_{1}^{b-a}: C(j) \rightarrow C(k)
$$

satisfies $\tau_{2}^{-1} g \tau_{1}^{b-a} \in T^{*}(a, b)$ and hence

$$
\tau_{2}^{-1} g \tau_{1}^{b-a}(2 b-a)=3^{\prime} \text {. }
$$

But this implies $g(3 b-2 a)=4^{\prime}$. Proceeding inductively by using the maps $\tau_{2}^{-(r-1)} g \tau_{1}^{(r-1)(b-a)}$ one then shows that $g(a+(r+1)(b-a))=r+2^{\prime}$ for all $r$, and hence $g=T(a, b)$ as required. In practice, the proof of $g^{-1}\left(3^{\prime}\right)=2 b-a$ often proceeds by considering all possibilities for $g^{-1}\left(3^{\prime}\right)$ subject to the hypotheses that $g$ is simplicial and $C(j), C(k) \in C \backslash C^{\prime}$, and eliminating all of them except $g^{-1}\left(3^{\prime}\right)=2 b$ $-a$. The required result follows since then either $g^{-1}\left(3^{\prime}\right)=2 b-a$ as desired, or $g^{-1}\left(3^{\prime}\right) \neq 2 b-a$ in which case the hypothesis $C(k) \in C \backslash C^{\prime}$ must be violated.

There are, of course, six possible sets of mappings $T^{*}(a, b)$ to consider. We carry out the details here only for the case involving $T^{*}(1, j)$, the other cases being essentially similar computations. A full treatment of all the cases may be found in [11].

Suppose then that $f \in T^{*}(1, j)$. Thus we have $f(1)=1^{\prime}, f(j)=2^{\prime}$, and $f(2)=k^{\prime}$. Our first step will be to show that $f^{-1}\left(k-1^{\prime}\right)=n+3-j$. We remark that since $\left\langle k-1^{\prime}, k^{\prime}, 1^{\prime}\right\rangle \notin C(k)_{(2)}$ by Lemma 2.1 , it follows that $f^{-1}\left\langle k-1^{\prime}, k^{\prime}, 1^{\prime}\right\rangle \notin C(j)_{(2)}$ since $f$ is an isomorphism.

It is convenient to consider first the case $n=2 j-1$. This condition yields $f^{-1}\left(k-1^{\prime}\right) \in\{j+1, j+2, n\}$. If $f^{-1}\left(k-1^{\prime}\right)=n$, then $\langle n, 2\rangle=f^{-1}\left\langle k-1^{\prime}, k^{\prime}\right\rangle$ $\in C(j)_{(1)}$, so that combining with $C(j) \notin C^{\prime}$ we get $j=4$. This gives $n=7$, contradicting $C(j) \notin C^{\prime}$. Suppose then that $f^{-1}\left(k-1^{\prime}\right)=j+1$. Then we have $\left\langle 2^{\prime}, k-1^{\prime}\right\rangle=f\langle j, j+1\rangle \in C(k)_{(1)}$, and it follows that $k=4$. Now by preservation of cells we have $\left\langle f^{-1}\left(n^{\prime}\right), 1, j+1\right\rangle=f^{-1}\left\langle n^{\prime}, 1^{\prime}, k-1^{\prime}\right\rangle \in C(j)_{(2)}$, so using $n=2 j$ -1 it follows that $f^{-1}\left(n^{\prime}\right)=j+2$. But now $k=4$ gives $\langle j, j+2\rangle=f\left\langle n^{\prime}, 2^{\prime}\right\rangle \in$ $C(j)_{(1)}$, and thus $j=4$. We then have the contradiction $n=7$ and $C(j) \in C^{\prime}$. Finally assume that $f^{-1}\left(k-1^{\prime}\right)=j+2$. Arguing as above we get $f^{-1}\left(n^{\prime}\right)=j+1$. Now $\left\langle n^{\prime}, 2^{\prime}\right\rangle=f\langle j+1, j\rangle \in C(k)_{(1)}$ so we have $k=4$. But this implies $k-1^{\prime}=3^{\prime}$ and $\langle j, j+2\rangle=f^{-1}\left\langle 2^{\prime}, 3^{\prime}\right\rangle \in C(j)_{(1)}$ so that $j=4$. It follows again that $n=7$ and $C(j) \in C^{\prime}$, a contradiction. We have thus shown that no isomorphism $f \in T^{*}(1, j)$ yields an image plex $C(k) \in C \backslash C^{\prime}$ when $n=2 j-1$.

We may henceforth assume that $n \neq 2 j-1$. It follows that $f^{-1}\left(k-1^{\prime}\right) \in\{n+3$ $-j, n\}$ if $j=4$, while $f^{-1}\left(k-1^{\prime}\right)=n+3-j$ exclusively if $j \neq 4$. Suppose then that $j=4$ and $f^{-1}\left(k-1^{\prime}\right)=n$. In order to preserve cells we have $\left\langle f^{-1}\left(n^{\prime}\right), 1, n\right\rangle$ $=f^{-1}\left\langle n^{\prime}, 1^{\prime}, k-1^{\prime}\right\rangle \in C(j)_{(2)}$ so we get $f^{-1}\left(n^{\prime}\right)=j-1$. This gives $\left\langle n^{\prime}, 2^{\prime}\right\rangle=$ $f\langle j-1, j\rangle \in C(k)_{(1)}$ and hence $k=4$. It follows that $\langle j, n\rangle=f^{-1}\left\langle 2^{\prime}, 3^{\prime}\right\rangle \in C(j)_{(1)}$. But combining $n \neq 2 j-1$ with $C(j) \in C \backslash C^{\prime}$ we get $n \geqslant 2 j+1$ and hence $\langle j, n\rangle \notin C(j)_{(1)}$, a contradiction. We are thus reduced to $f^{-1}\left(k-1^{\prime}\right)=n+3-j$, as desired. We also note that since $\left\langle f^{-1}\left(n^{\prime}\right), 1, n+3-j\right\rangle=f^{-1}\left\langle n^{\prime}, 1^{\prime}, k-1^{\prime}\right\rangle \in$ $C(j)_{(2)}$, we also get $f^{-1}\left(n^{\prime}\right)=n+2-j$. 
We may now prove that $f=T(1, j)$. As has been previously observed, it suffices to show that $f^{-1}\left(3^{\prime}\right)=2 j-1$. This will be done using the following descending induction. It has been shown that $f^{-1}\left(k-1^{\prime}\right)=n+3-j=f^{-1}\left(k^{\prime}\right)-(j-1)$ and $f^{-1}\left(n^{\prime}\right)=n+2-j=f^{-1}\left(1^{\prime}\right)-(j-1)$. Let us then assume that for some integer $R$ satisfying $3<R<k$, we have proved that $f^{-1}\left(r^{\prime}\right)=f^{-1}\left(r+1^{\prime}\right)-(j-1)$ for all $r$ in the ranges $R \leqslant r<k$ and $R-k+1 \leqslant r<1$. Our object is to show that $f^{-1}\left(R-1^{\prime}\right)=f^{-1}\left(R^{\prime}\right)-(j-1)$. Let $m=(k-R)(1-j)+1$ for brevity. Then we have $f^{-1}\left(R-1^{\prime}\right)=N\left(f^{-1}\left(R^{\prime}\right)\right) \cap N\left(f^{-1}\left(R-k+1^{\prime}\right)\right)=N(m) \cap N(m+1)$. Since $n \geqslant 2 k-1$ we have $\left\langle R-1^{\prime}, R^{\prime}, R-k+1^{\prime}\right\rangle \notin C(k)_{(2)}$ and hence

$$
f^{-1}\left\langle R-1^{\prime}, R^{\prime}, R-k+1^{\prime}\right\rangle \notin C(j)_{(2)} \text {. }
$$

Thus $f^{-1}\left(R-1^{\prime}\right) \neq m+j-1$, so it follows (using $\left.n \neq 2 j-1\right)$ that $f^{-1}\left(R-1^{\prime}\right)=$ $m-(j-2)$. But this yields $f^{-1}\left(R-1^{\prime}\right)=m-(j-2)=1+(k-R)(1-j)=2$ $-(k-R+1)(j-1)=f^{-1}\left(R^{\prime}\right)-(j-1)$. Our induction is completed and we get the desired result $f^{-1}\left(3^{\prime}\right)=2 j-1$.

We are now ready for the characterization of those complexes $C(n, j) \in C$ satisfying $\Gamma_{2}(C(n, j)) \cong \mathbf{Z}_{n}$. We write $C(j)$ for $C(n, j)$ in the proof since $n$ is fixed.

Theorem 3. Let $C(n, j) \in C$. Then we have $\Gamma_{2}(C(n, j)) \cong \mathbf{Z}_{n}$ if and only if $C(n, j) \in C \backslash C^{\prime}$.

Proof. We already know from Theorem 1 that if $C(j) \in C^{\prime}$, then $\Gamma_{2}(C(j)) \neq \mathbf{Z}_{n}$. Hence necessity is proved.

Suppose now that $C(j) \in C \backslash C^{\prime}$, and assume to the contrary that $\Gamma_{2}(C(j)) \not \mathbf{Z}_{n}$. Then there exists an automorphism $\sigma \in \Gamma_{0}(C(j))$ such that $\sigma \notin\langle\tau\rangle$, where $\tau=(12$ $\cdots n)$. Thus $\sigma$ is an isomorphism $\sigma: C(j) \rightarrow C(j)$ which is not a power of $T(1,2)$.

By Theorem 2 we know that $\sigma$ is a division isomorphism. Thus $\sigma=T(a, b)$ for some ordered pair $(a, b)$, and we consider each possibility for $(a, b)$ in turn.

Suppose first that $\sigma=T(1, j)$. By definition $\sigma(1)=1, \sigma(j)=2$, and $\sigma(2)=j$, so that we must have $(j-1)^{2} \equiv 1(\bmod n)$. When $n=p^{\alpha} \geqslant 9$ with $p$ odd the two solutions to this congruence are $j \equiv 0$ and $2(\bmod n)$, neither of which is permitted for a plex $C(j) \in C$. When $n=2^{\alpha} \geqslant 16$ there are four solutions, $j=0,2, n / 2+2$, and $n / 2$. The first three are disallowed by definition of $C$ while the last implies that $C(j) \in C^{\prime}$, as required. Next suppose $\sigma=T(j, 1)$. Since $\sigma(j)=1, \sigma(1)=2$, and $\sigma(2)=j$, we must have $(1-j)(j-1) \equiv 2-j(\bmod n)$. Thus $j^{2}-3 j+3 \equiv 0$ $(\bmod n)$, and we get $C(j) \in C^{\prime}$. Assume now that $\sigma=T(2, j)$. Using $\sigma(2)=1$, $\sigma(j)=2$, and $\sigma(1)=j$, we find that $(j-2)(j-1) \equiv-1(\bmod n)$, and again we get $j^{2}-3 j+3 \equiv 0(\bmod n)$ leading to $C(j) \in C^{\prime}$. Next assume $\sigma=T(j, 2)$. Since $\sigma(j)=1, \sigma(2)=2$, and $\sigma(1)=j$, we get $(2-j)(j-1) \equiv 1-j(\bmod n)$ and thus $(j-3)(j-1) \equiv 0(\bmod n)$. Writing $n=p^{r}$ for prime $p$ and using $n>2 j-2>j$ -1 we find that $p \mid j-3$ and $p \mid j-1$. It follows that $p=2$ so $n=2^{r}$. Since $C(j) \in C \backslash C^{\prime}$ and $n$ is even we must have $n \geqslant 2 j+2$ and thus $j<2^{r-1}$. Now observe that one of $j-1$ and $j-3$ has congruence class 2 modulo 4 , and it follows that the largest power of 2 dividing $(j-1)(j-3)$ is at most $2^{r-1}$. This contradicts $n \mid(j-1)(j-3)$, and the theorem is proved. 
We now turn our attention to the problem of determining the number of isomorphism types of plexes $C(n, j) \in C$ satisfying $\Gamma_{2}(C(n, j)) \cong \mathbf{Z}_{n}$. We will call this number $I(n)$. The following lemma is useful in the determination of $I(n)$.

LEMMA 4.1. Let $C(j) \in C \backslash C^{\prime}$.

(1) For any $\alpha \in T^{j}, \alpha \neq T(1,2)$, if $\alpha(C(j))=C(k) \in C$, then $k \neq j$.

(2) For any two distinct $\alpha, \beta \in T^{j}$, if $\alpha(C(j))=C\left(k_{1}\right)$ and $\beta(C(j))=C\left(k_{2}\right)$ with $C\left(k_{1}\right), C\left(k_{2}\right) \in C$, then $k_{1} \neq k_{2}$.

Proof. We will make use of Table 1 which gives the value of $k$ satisfying $T(a, b)[C(j)]=C(k)$ for any $T(a, b) \in T^{j}$.

For statement (1), let us assume the contrary and then consider each possible $\alpha$ in turn. We need not consider $\alpha=T(2,1)$ since $T(2,1)[C(j)]=C(n+3-j) \notin C$. Suppose then that $\alpha=T(1, j)$. By Table 1 we must have $j \equiv(j-1)^{-1}+1(\bmod n)$, implying that $j(j-2) \equiv 0(\bmod n)$. Since $n$ is a prime power and $n>j$ it follows that $n=2^{r}$. The condition $n \geqslant 2 j-3$ and the congruence above imply that $n=2 j$, contradicting $C(j) \in C \backslash C^{\prime}$. Now suppose $\alpha=T(j, 1)$. The contrary assumption forces $j=n+3-(j-1)^{-1}-1(\bmod n)$, which immediately leads to the contradiction $j^{2}-3 j+3 \equiv 0(\bmod n)$. Next assume that $\alpha=T(2, j)$, so that our assumption gives $j \equiv(j-2)^{-1}+1(\bmod n)$. Again we get $j^{2}-3 j+3 \equiv 0(\bmod n)$, a contradiction. Finally if $\alpha=T(j, 2)$, then $j \equiv n+3+(j-2)^{-1}-1(\bmod n)$. This leads to $(j-3)(j-1) \equiv 0(\bmod n)$, implying that $n=2^{r}$. Now combining the congruence with $n \geqslant 2 j+2$ we find that $2^{r}$ does not divide $(j-1)(j-3)$, a contradiction.

In proving statement (2), we will again assume the contrary and then consider all possible pairs $\alpha, \beta \in T^{j}$. We may immediately eliminate pairs $T(a, b), T(b, a)$ from consideration since not both $T(a, b)[C(j)]$ and $T(b, a)[C(j)]$ are members of $C$. In addition we may assume that neither $\alpha$ nor $\beta$ is $T(1,2)$ or $T(2,1)$ since $T(1,2)[C(j)]$ $=C(j)$ thereby reducing to statement $(1)$, while $T(2,1)[C(j)]=C(n+3-j) \notin C$.

Let us then consider the remaining pairs $\alpha, \beta$ in turn. Suppose $\alpha=T(1, j)$ and $\beta=T(2, j)$. Our contrary assumption and Table 1 yield

$$
(j-1)^{-1}+1 \equiv-(j-2)^{-1}+1 \quad(\bmod n),
$$

which gives $2 j-3 \equiv 0(\bmod n)$, contradicting $C(j) \notin C^{\prime}$. Now suppose $\alpha=T(1, j)$ and $\beta=T(j, 2)$, so that $(j-1)^{-1}+1 \equiv n+3+(j-2)^{-1}-1(\bmod n)$. This leads to the contradiction $j^{2}-3 j+3 \equiv 0(\bmod n)$. If $\alpha=T(j, 1)$ and $\beta=T(2, j)$, then $n+3-(j-1)^{-1}-1 \equiv-(j-2)^{-1}+1(\bmod n)$ and again we get the contradiction $j^{2}-3 j+3 \equiv 0(\bmod n)$. Finally if $\alpha=T(j, 1)$ and $\beta=T(j, 2)$, then $n+3-(j-1)^{-1}-1 \equiv n+3+(j-2)^{-1}-1$ and we arrive at the contradiction $2 j-3 \equiv 0(\bmod n)$. The lemma is proved.

For convenience, we make the following definitions. Let $Q(n)$ be the set of integers $j$ for which $C(n, j) \in C$ and $\Gamma_{2}(C(n, j)) \cong \mathbf{Z}_{n}$, and let $Y(n)$ be the set of integers $j$ for which $C(n, j) \in C$ and $\Gamma_{2}(C(n, j)) \neq \mathbf{Z}_{n}$. For $j \in Q(n)$ define $\lambda(j)$ to be the number of plexes $C(n, k), k \in Q(n)$, in the same isomorphism class as 
$C(n, j)$. Finally, for reals $r$ and $s$ denote by $[r, s]$ the set of integers $n$ satisfying $r \leqslant n \leqslant s$. Thus we have the partition $[1,\{n / 2\}+1]=Q(n) \cup Y(n)$.

It is now possible to calculate $I(n)$.

THEOREM 4. Let $n \geqslant 9$ with $n=p^{\alpha}$ for $p$ prime, and let $m=\{n / 2\}$. Then the number $I(n)$ of isomorphism classes among the set of plexes $C(n, j) \in C$ satisfying $\Gamma_{2}(C(n, j)) \cong \mathbf{Z}_{n}$ (equivalently $\left.C(n, j) \in C \backslash C^{\prime}\right)$ is given by

$$
I(n)= \begin{cases}{\left[\frac{m+1}{p}\right]+\frac{1}{3}\left(m-2\left[\frac{m+1}{p}\right]-1\right)-1} & \begin{array}{l}
\text { when } p \equiv 1(\bmod 6) \\
\text { or } p=3,
\end{array} \\
{\left[\frac{m+1}{p}\right]+\frac{1}{3}\left(m-2\left[\frac{m+1}{p}\right]\right)-1} & \text { when } p \equiv 5(\bmod 6), \\
\frac{n}{4}-2 & \text { when } p=2 .\end{cases}
$$

Proof. Our method is to use Theorem 2 to identify the isomorphism classes of complexes $C(n, j) \in C$ with the orbits of $C$ under the action of the group of division isomorphisms.

Let us begin with the determination of the numbers $\lambda(j)$ for $j \in Q(n)$. By Theorem 2 two distinct complexes $C(j), C(k) \in C$ are isomorphic if and only if they are division isomorphic, say, by $f: C(j) \rightarrow C(k)$. Setting

$$
T=T^{j} \backslash\{T(1,2), T(2,1)\},
$$

we may assume that $f \in T$ since $j \neq k$ and $j, k \leqslant\{n / 2\}+1$. Thus we have $f \in$ $\{T(1, j), T(j, 1), T(2, j), T(j, 2)\}$. Now observe that $T(a, b)[C(j)]=C(k)$ if and only if $T(b, a)[C(j)]=C(n+3-k)$. Hence there are at most two isomorphisms in $T$ whose actions on $C(j)$ yield plexes in $C$, and thus there are at most two plexes $C\left(k_{1}\right), C\left(k_{2}\right) \in C$ distinct from $C(j)$ and in the same isomorphism class as $C(j)$. It follows that $\lambda(j) \leqslant 3$ for all $j \in Q(n)$. Furthermore, from the definition of $T(a, b)$ we have an isomorphism $T(a, b)$ defined only when $|a-b|$ and $n=p^{\alpha}$ are relatively prime. Thus we have the upper bounds $\lambda(j) \leqslant 3$ if $j \neq 1$ or $2(\bmod p)$ and $\lambda(j) \leqslant 2$ if $j \equiv 1$ or $2(\bmod p)$. We now prove that the corresponding lower bounds also hold, from which equality follows. Suppose first that $j \neq 1$ or $2(\bmod p)$. Then all isomorphisms in $T$ are defined on $C(j)$, and from the remarks above precisely two of them, say $T(a, b)$ and $T(c, d)$ with $|a-b| \neq|c-d|$, yield isomorphic images of $C(j)$ which are in $C$. Calling these images $C\left(k_{1}\right)$ and $C\left(k_{2}\right)$ as above, we appeal to Lemma 4.1 to conclude that the three integers $j, k_{1}$, and $k_{2}$ are distinct, and thus $\lambda(j)=3$. If $j \equiv 1$ or $2(\bmod p)$, then precisely one of the $j-1$ and $j-2$ is relatively prime to $n$, and as above there is one isomorphism $\beta \in T$ such that $\beta(C(j))=C(k) \in C$. Again by Lemma 4 .1 we have $j \neq k$ and so $\lambda(j)=2$. To summarize then, it has been shown that for $j \in Q(n)$ we have $\lambda(j)=3$ if $j \neq 1$ or 2 $(\bmod p)$, while $\lambda(j)=2$ if $j \equiv 1$ or $2(\bmod p)$.

In view of this result, it is useful to adopt the following notation. For $r=2$ and 3 define $Q_{r}(n)$ as the set of integers $j \in Q(n)$ for which $\lambda(j)=r$, and $I_{r}(n)$ as the 
number of isomorphism classes among the set of plexes $C(j)$ with $j \in Q_{r}(n)$. Thus we have the partitions $Q(n)=Q_{2}(n) \cup Q_{3}(n),[1,\{n / 2\}+1]=Q_{2}(n) \cup Q_{3}(n) \cup$ $Y(n)$, and the equality $I(n)=I_{2}(n)+I_{3}(n)$.

We may now proceed to the calculation of $I(n)$. Suppose first that $n=p^{\alpha}$ where $p \equiv 1(\bmod 6)$. Let $f(x)=x^{2}-x+3$ and observe that if $r$ is a solution to $f(x) \equiv 0$ $(\bmod n)$, then so is $n+3-r$. Since these two must be all the solutions, it follows that there is precisely one integer, call it $r$, satisfying $r \in[1,\{n / 2\}+1]$ and $f(r) \equiv 0(\bmod n)$. Now none of $1,2,3$, and $\{n / 2\}+1$ satisfy $f(x) \equiv 0(\bmod n)$ so that we may conclude by Theorem 3 that $Y(n)=\{1,2,3,\{n / 2\}+1, r\}$. Let $X$ be the set of integers $j$ satisfying $p<j \leqslant\{n / 2\}+1$ and $j \equiv 1$ or $2(\bmod p)$. We claim $Q_{2}(n)=X$. From the discussion above we know that $Q_{2}(n) \subset X$. For the opposite inclusion, note that if $j \in X$, then not both $j-1$ and $j-2$ are relatively prime to $n$. Hence we have $X \cap Q_{3}(n)=\varnothing$. It therefore remains to show that $X \cap Y(n)=\varnothing$. This amounts to verifying that $\{n / 2\}+1 \notin X$ and $r \notin X$. Since $p>9$ it is obvious that $\{n / 2\}+1 \neq 1$ or $2(\bmod p)$. To show $r \notin X$, observe that since $f(r) \equiv 0$ $\left(\bmod p^{\alpha}\right)$, we have $f(r) \equiv 0(\bmod p)$. But if $r \equiv 1$ or $2(\bmod p)$, then $f(r) \equiv 1$ $(\bmod p)$, a contradiction. Now by definition of $X$ and since $\{n / 2\}+1 \neq 0(\bmod p)$, we have $\left|Q_{2}(n)\right|=|X|=2[(\{n / 2\}+1) / p]$, and thus

$$
I_{2}(n)=\frac{1}{2}\left|Q_{2}(n)\right|=[(\{n / 2\}+1) / p] .
$$

Now we also get $\left|Q_{3}(n)\right|=|Q(n)|-\left|Q_{2}(n)\right|=\{n / 2\}+1-|Y|-\left|Q_{2}(n)\right|=$ $\{n / 2\}-2[(\{n / 2\}+1) / p]-4$. Thus $I_{3}(n)$ is given by $I_{3}(n)=\frac{1}{3}\left|Q_{3}(n)\right|$, and combining this with $I(n)=I_{2}(n)+I_{3}(n)$ we get the required result.

Suppose $p=3$. Here we find that $Y(n)$ is $\{1,2,3,\{n / 2\}+1\}$ so that $Q(n)=$ $\{n / 2\}+1-|Y(n)|=\{n / 2\}-3$. Defining $X$ as above (with $p=3$ ) we have $|X|=2([(\{n / 2\}+1) / 3]-1)$ since $\{n / 2\}+1 \equiv 0(\bmod 3)$ when $n=3^{\alpha}$, and it is similarly proved that $Q_{2}(n)=X$. Thus we get $\left|Q_{3}(n)\right|=|Q(n)|-\left|Q_{2}(n)\right|=\{n / 2\}$ $-2[(\{n / 2\}+1) / 3]-1$. By definition of $I_{r}(n), r=2,3$, we therefore obtain

$$
I_{2}(n)=\frac{1}{2}\left|Q_{2}(n)\right|=[(\{n / 2\}+1) / 3]-1
$$

and

$$
I_{3}(n)=\frac{1}{3}\left|Q_{3}(n)\right|=\frac{1}{3}(\{n / 2\}-2[(\{n / 2\}+1) / 3]-1),
$$

from which the formula follows.

Now assume $p \equiv 5(\bmod 6)$. We find that

$$
Y(n)=\{1,2,3,\{n / 2\}+1\}, \quad|X|=2[(\{n / 2\}+1) / p]
$$

since $\{n / 2\}+1 \neq 0,1$, or $2(\bmod n)$, and as above $Q_{2}(n)=X$. Thus

$$
\left|Q_{3}(n)\right|=|Q(n)|-\left|Q_{2}(n)\right|=\{n / 2\}-3-2[(\{n / 2\}+1) / p],
$$

and we get

$$
\begin{aligned}
I(n) & =I_{2}(n)+I_{3}(n)=\frac{1}{2}\left|Q_{2}(n)\right|+\frac{1}{3}\left|Q_{3}(n)\right| \\
& =[(\{n / 2\}+1) / p]+\frac{1}{3}(\{n / 2\}-2[(\{n / 2\}+1) / p])-1,
\end{aligned}
$$

as required. 
Finally consider the case $p=2$. By Theorem 3 the exceptional set $Y(n)$ is $\{1,2,3, n / 2, n / 2+1\}$, and we get $|Q(n)|=n / 2+1-|Y(n)|=n / 2-4$. Since $p=2$, we also get $Q(n)=Q_{2}(n)$ and $Q_{3}(n)=\varnothing$. It follows that

$$
I(n)=\frac{1}{2}\left|Q_{2}(n)\right|=n / 4-2,
$$

and the theorem is proved.

COROllary 4.1. If $p^{\alpha} \geqslant 9$, then we have $M_{1}^{(2)}\left(\mathbf{Z}_{p^{\alpha}}\right)=M_{0}^{(2)}\left(\mathbf{Z}_{p^{\alpha}}\right)=p^{\alpha}$.

Proof. We give the proof only for $M_{1}^{(2)}\left(\mathbf{Z}_{p^{\alpha}}\right)$, as that for $M_{0}^{(2)}\left(\mathbf{Z}_{p^{\alpha}}\right)$ is obtained by replacing cells by points and cell groups by point groups throughout.

The upper bound $M_{1}^{(2)}\left(\mathbf{Z}_{p^{\alpha}}\right) \leqslant p^{\alpha}$ follows from the existence, guaranteed by Theorem 4 , of a plex $C\left(p^{\alpha}, j\right)$ satisfying $\Gamma_{2}\left(C\left(p^{\alpha}, j\right)\right) \cong \mathbf{Z}_{p^{\alpha}}$ and $\left|C\left(p^{\alpha}, j\right)_{(2)}\right|=p^{\alpha}$ for $p^{\alpha} \geqslant 9$.

For the lower bound, let $K$ be any plex satisfying $\Gamma_{2}(K) \cong \mathbf{Z}_{p^{\alpha}}$, and let $\sigma$ be a generator of $\Gamma_{2}(K)$. Clearly $|\sigma|=p^{\alpha}$, and $|\sigma|$ is the least common multiple of the lengths of the cycles appearing in the disjoint cycle decomposition of $\sigma$. Hence a $p^{\alpha}$-cycle appears in this decomposition, so that $\left|K_{(2)}\right| \geqslant p^{\alpha}$.

Let us now observe that the complex $C(n, j)$ can also be defined when $n$ is not a prime power. We can then ask the same question considered above. For fixed $n$, what is the number $I(n)$ of isomorphism classes of complexes $C(n, j)$ satisfying $\Gamma_{2}(C(n, j)) \cong \mathbf{Z}_{n}$ ? We give some values of $I(n)$ for small $n$.

TheOREM 5. $I(10)=1, I(12)=2, I(14)=2, I(15)=2$.

We have shown above that $M_{1}^{(2)}\left(\mathbf{Z}_{p^{\alpha}}\right)=p^{\alpha}$, and that the minimum was achieved by the complexes $C\left(p^{\alpha}, j\right)$ satisfying $\Gamma_{2}\left(C\left(p^{\alpha}, j\right)\right) \cong \mathbf{Z}_{p^{\alpha}}$. These $C\left(p^{\alpha}, j\right)$ were characterized in Theorem 3 (using the parameters $p^{\alpha}$ and $j$ ) as the ones belonging to the set $C \backslash C^{\prime}$. Now clearly the group $\Gamma_{2}\left(C\left(p^{\alpha}, j\right)\right)$ contains an element $\tau$ whose cycle structure (as a permutation on $\left.C\left(p^{\alpha}, j\right)_{(2)}\right)$ consists entirely of a $p^{\alpha}$-cycle. Indeed, $\tau$ is the permutation on $C\left(p^{\alpha}, j\right)_{(2)}$ which sends cell $\langle i, i+1, i+j-1\rangle$ to cell $\langle i+1, i+2, i+j\rangle$ (indices read modulo $p^{\alpha}$ ). Our next objective is to state a theorem (without proof for brevity) which characterizes the set of all 2-complexes $K$ such that $\left|K_{(2)}\right|=p^{\alpha}$ and there exists $\sigma \in \Gamma_{2}(K)$ whose cycle structure (as a permutation on $K_{(2)}$ ) consists entirely of a $p^{\alpha}$-cycle. The main consequence of this characterization will be that the realization of $M_{1}^{(2)}\left(\mathbf{Z}_{p^{\alpha}}\right)$ by $C\left(p^{\alpha}, j\right)$ is unique. That is, if a 2-complex $K$ satisfies $\left|K_{(2)}\right|=p^{\alpha}$ and $\Gamma_{2}(K) \cong \mathbf{Z}_{p^{\alpha}}$, then $K=C\left(p^{\alpha}, j\right)$ for some $j$.

First we fix some notation. Let $K$ be a 2-complex such that $\left|K_{(2)}\right|=n$ with $n=p^{\alpha}$ a prime power, and such that there exists $\sigma \in \Gamma_{2}(K)$ which is an $n$-cycle. Thus we may write $\sigma=\left(a_{1} a_{2} \cdots a_{n}\right)$ where the $a_{i}$ are all the 2-cells of $K$. Such a plex will be called $n$-permutable. If $c$ is any cycle appearing in the disjoint cycle structure of some element of $\Gamma_{2}(K)$, we denote by $K(c)$ the complex $\left\langle a_{1}, a_{2}, \ldots, a_{n}\right\rangle$ induced by the cells appearing in $c$. Now let $K$ be $n$-permutable and let $d$ satisfy $(d, n)>1$, or, 
equivalently, $(d, p)>1$. Then $\sigma^{d}$ will have a disjoint cycle decomposition $\sigma^{d}=$ $\prod_{i=1}^{(n, d)} c_{i}$ where $c_{i}$ is a cycle of length $n /(n, d)$. It is clear that the complexes $K\left(c_{i}\right)$, $1 \leqslant i \leqslant(n, d)$, are mutually isomorphic and that $K$ is the cell disjoint union of these complexes. Letting $K\left(c_{i}\right)=K^{\prime}$, we will say that $K$ is a $K^{\prime}$-superposition or $K$ is a superposition of $K^{\prime}$. If $(d, n)=1$, then $\sigma^{d}$ is an $n$-cycle, and we have $K\left(\sigma^{d}\right)=K(\sigma)$ $=K$. We will then continue to call $K$ a $K\left(\sigma^{d}\right)$-superposition. Observe that $K$ may be both a $K^{\prime}$-superposition and a $K^{\prime \prime}$-superposition for nonisomorphic $K^{\prime}$ and $K^{\prime \prime}$ (via different powers of $\sigma$ of course). If $K$ satisfies $\Gamma_{2}(K) \cong \mathbf{Z}_{n}$ in addition to being $n$-permutable then we will say that $K$ is $n$-cyclic. If $G$ is a graph, denote by $P(G)$ the 2-complex having $V(G)$ as its points and the triangles of $G$ as its 2-simplexes. We now define several special classes of plexes, some of which were first introduced in [5] by Harary and Duke in their investigation of the Ramsey numbers of plexes. As usual, the point set of a plex $K$ will be denoted by $V(K)$ and the set of 2-cells by $K_{(2)}$. We now define the $n$-book $B_{n}$, the $(n, 0)$-cycle $C_{n, 0}$, the $(d, n / d)$ book-cycle $B C(d, n / d)$, the $d$-identified $n$-cycle ${ }_{d} C_{n}$, the $n$-star $S_{n}$, and the $n$-disk $D_{n}$, as follows:

(1) The $n$-book:

$$
\begin{aligned}
V\left(B_{n}\right) & =\{x, y, 1,2, \ldots, n\}, \\
B_{n}(2) & =\{\langle x, y, i\rangle: 1 \leqslant i \leqslant n\} .
\end{aligned}
$$

(2) The $(n, 0)$-cycle:

$$
\begin{aligned}
V\left(C_{n, 0}\right) & =\left\{1,2, \ldots, n, 1^{\prime}, 2^{\prime}, \ldots, n^{\prime}\right\}, \\
C_{n, 0(2)} & =\left\{\left\langle i, i+1, i^{\prime}\right\rangle: 1 \leqslant i \leqslant n, \text { integers read modulo } n\right\} .
\end{aligned}
$$

(3) The $(d, n / d)$ book-cycle:

$$
\begin{aligned}
V(B C(d, n / d)) & =\left\{1,2, \ldots, d, 1_{i}, 2_{i}, \ldots, d_{i}: 1 \leqslant i \leqslant n / d\right\}, \\
B C(d, n / d)_{(2)} & =\left\{\left\langle k, k+1, k_{i}\right\rangle: 1 \leqslant i \leqslant n / d, 1 \leqslant k \leqslant d\right\} .
\end{aligned}
$$

(4) The $d$-identified $n$-cycle:

$$
\begin{aligned}
V\left({ }_{d} C_{n}\right) & =\left\{1,2, \ldots, n, 0^{\prime}, 1^{\prime}, \ldots,(d-1)^{\prime}\right\}, \quad d \text { a divisor of } n, \\
{ }_{d} C_{n(2)} & =\left\{\left\langle i, i+1, i^{\prime}\right\rangle: 1 \leqslant i \leqslant n, i \equiv i^{\prime}(\bmod d)\right\} .
\end{aligned}
$$

(5) The $n$-star:

$$
\begin{aligned}
V\left(S_{n}\right) & =\left\{\Omega, 1,2, \ldots, n, 1^{\prime}, 2^{\prime}, \ldots, n^{\prime}\right\}, \\
S_{n(2)} & =\left\{\left\langle\Omega, i, i^{\prime}\right\rangle: 1 \leqslant i \leqslant n\right\} .
\end{aligned}
$$

(6) The $n$-disk:

$$
\begin{aligned}
V\left(D_{n}\right) & =\{1,2, \ldots, n, \Omega\}, \\
D_{n(2)} & =\{\langle\Omega, i, i+1\rangle: 1 \leqslant i \leqslant n\} .
\end{aligned}
$$

An example of a plex in each of the classes defined above is given in Figure 2.1. 


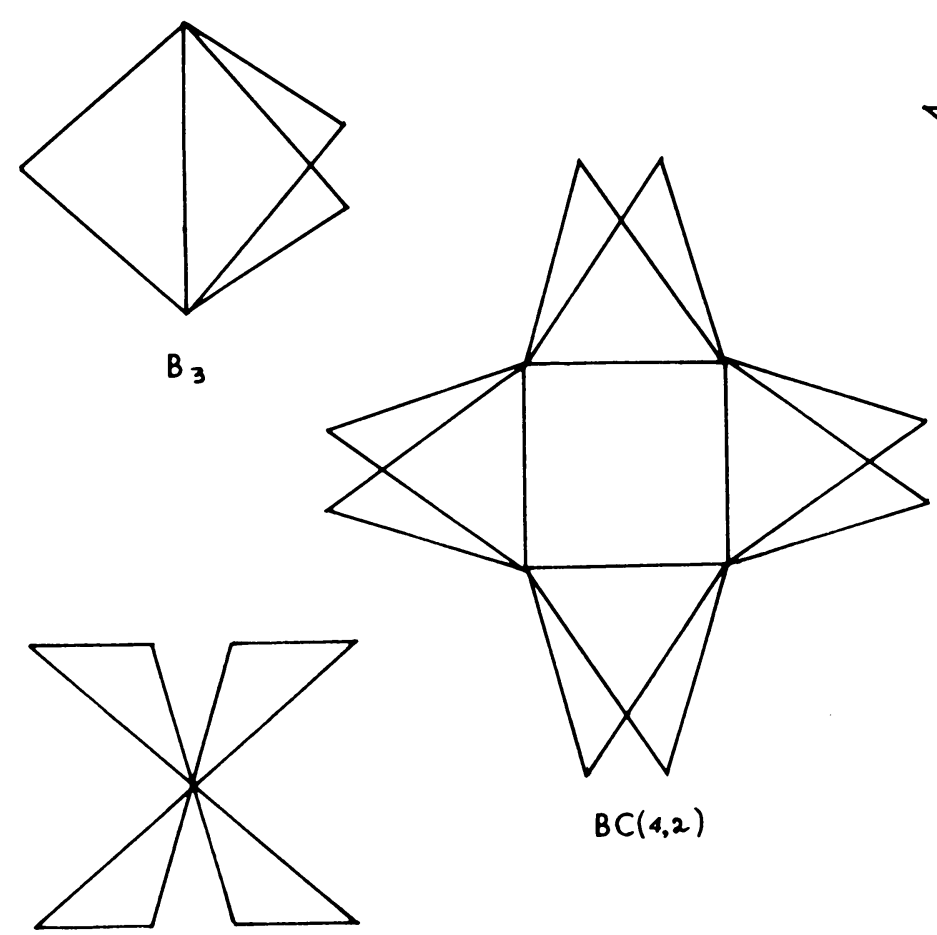

$\mathrm{S}_{4}$

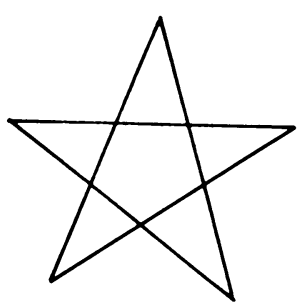

$C_{5,0}$

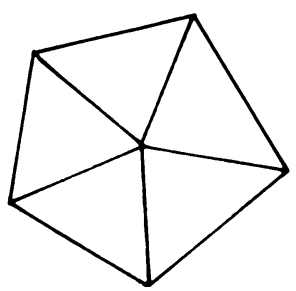

$D_{5}$

Figure 2.1. Examples of special classes of plexes

We are now ready for the first step, Theorem 6 , of our characterization. The proof is lengthy and is omitted for brevity.

THEOREM 6. Let $r$ range over divisors of $n$. Then a 2-complex $K$ is n-permutable if and only if it is a superposition of one of the following complexes.

(a) $B_{r}$,

(b) $C_{r, 0}$,

(c) $D_{r}$,

(d) $S_{r}$,

(e) $C(r, j)$,

(f) $r P K_{3}$.

Theorem 6 may be used to derive the following structural characterization of all $n$-permutable plexes for $n$ a prime power. The proof of this result involves a lengthy computational argument which we omit here. Details may be found in [11].

For the statement of this characterization we require the following notation. Recall that for any graph $G$, we denote by $P(G)$ the 2-dimensional simplicial complex whose points are those of $G$ and whose 2-cells are the triangles in $G$ while the 1-cells are the edges of $G$. If $G$ and $H$ are graphs, we denote by $G+H$ (borrowing the notation of [4]) the graph whose point set is $V(G) \cup V(H)$ and whose edge set is $E(G) \cup E(H) \cup\{v w: v \in V(G), w \in V(H)\}$. The graph $K_{1}$ 

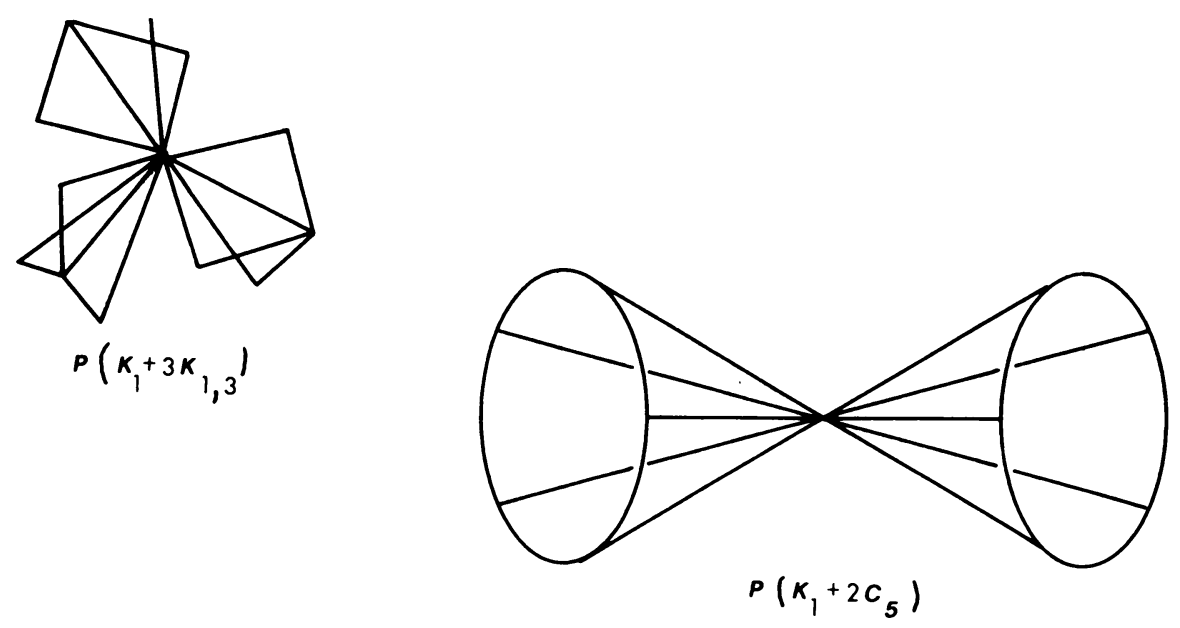

Figure 2.2. The complexes $P\left(K_{1}+3 K_{1,3}\right)$ and $P\left(K_{1}+2 C_{5}\right)$

consists of one point and no edges, $C_{n}$ is the cycle graph on $n$ points, $n G$ is the disjoint union of $n$ copies of the graph $G$, and $K_{1, r}$ is the graph $K_{1}+r K_{1}$. Illustrations of $P\left(K_{1}+3 K_{1,3}\right)$ and $P\left(K_{1}+2 C_{5}\right)$ are given in Figure 2.2.

Our characterization of $p^{\alpha}$-permutable plexes may now be given.

THEOREM 7. Let $n=p^{\alpha}$ be a prime power with $l, m$ positive integers such that $n / \operatorname{lm} \in \mathbf{Z}$. Then a 2-complex $K$ is n-permutable if and only if it is a disjoint union of copies of one of the following complexes.

(a) Some complex listed in Theorem 6,

(b) ${ }_{l} C_{l m}$,

(c) $B C(l, m)$,

(d) $P\left(K_{1}+l K_{1, m}\right)$

(e) $P\left(K_{1}+l C_{m}\right)$.

The following is an immediate consequence.

COROllaRY 7.1. For $p^{\alpha} \geqslant 9$, there is a unique 2-complex $K$ satisfying $\Gamma_{2}(K) \cong \mathbf{Z}_{p^{\alpha}}$ and $\left|K_{(2)}\right|=p^{\alpha}$, namely, $K=C\left(p^{\alpha}, j\right)$ for some $j$.

3. The values of $M_{1}^{(n)}(A)$ and $M_{0}^{(n)}(A)$. Let $A$ be an arbitrary finite abelian group. In this section we use the complexes analyzed in the previous sections and "cones" over these to form $n$-complexes, $n \geqslant 2$, that provide upper bounds for $M_{1}^{(n)}(A)$ and $M_{0}^{(n)}(A)$. It will be seen that for "almost all" groups $A$ these bounds are in fact the exact values of $M_{1}^{(n)}(A)$ and $M_{0}^{(n)}(A)$. In particular, we find the exact values of $M_{1}^{(n)}(A)$ for dimensions $n \geqslant 4$ when $A$ has no cyclic prime power direct factors of order less than 17, and for $n \geqslant 2$ when $A=\mathbf{Z}_{2}^{e(2)}$. We also find $M_{0}^{(2)}(A)$ for all $A$ except those having cyclic prime power factors of order less than 9 . These exact values and others are summarized in our main result, Theorem 11. 
We begin with some definitions. A finite abelian group $A$ will be written $A=\prod_{p, \alpha} \mathbf{Z}_{p^{\alpha}}^{e\left(p^{\alpha}\right)}$, where $e\left(p^{\alpha}\right)$ is the number of times a given $\mathbf{Z}_{p^{\alpha}}$ occurs as a factor in the canonical decomposition of $A$. We write $p^{\alpha} \mid A$ if $e\left(p^{\alpha}\right) \geqslant 1$, that is, if $\mathbf{Z}_{p^{\alpha}}$ appears in this decomposition. When $K$ is an $n$-complex satisfying $\Gamma_{n}(K) \cong A$ (resp. $\Gamma_{0}(K) \cong A$ ), then we say $K$ is an $n$-cell (resp. point) representation of $A$. If $n$ and $k$ are two positive integers, then $\delta_{k}(n)$ is defined to be 1 if $n \equiv 0(\bmod k)$ and 0 otherwise.

Some notation to be used in our constructions will now be introduced. Suppose $L$ and $K$ are two $n$-complexes with $v \in V(L)$ and $w \in V(K)$. We define the $n$-complex $v(L) \sim w(K)$ by $V(v(L) \sim w(K))=(V(L) \cup V(K)-\{v, w\}) \cup\{Z\}$, and $v(L)$ $\sim w(K)_{(n)}=\left\{\alpha \in L_{(n)}: v \notin \alpha\right\} \cup\left\{\alpha \in K_{(n)}: w \notin \alpha\right\} \cup\{(\alpha-v) \cup\{Z\}: v \in \alpha$, $\left.\alpha \in L_{(n)}\right\} \cup\left\{(\alpha-w) \cup\{Z\}: w \in \alpha, \alpha \in K_{(n)}\right\}$. Thus $v(L) \sim w(K)$ is obtained by attaching $K$ and $L$ together via the identification of $v$ and $w$. The new point $Z$ may be referred to as either $v$ or $w$. If $\left\{v_{i}\right\}_{i=1}^{n} \subset V(L)$ and $\left\{w_{i}\right\}_{i=1}^{n} \subset V(K)$ are sets of $n$ points in $L$ and $K$, then we define $v_{i}(L) \stackrel{1 \leqslant i \leqslant n}{\sim} w_{i}(L)$ recursively by

$$
\begin{aligned}
v_{i}(L) & \stackrel{1 \leqslant i \leqslant n}{\sim} w_{i}(K)=v_{n}\left(v_{i}(L) \stackrel{1 \leqslant i \leqslant n-1}{\sim} w_{i}(K)\right) \\
& \sim w_{n}\left(v_{i}(L) \stackrel{1 \leqslant i \leqslant n-1}{\sim} w_{i}(K)\right) .
\end{aligned}
$$

Thus $v_{i}(L) \stackrel{1 \leqslant i \leqslant n}{\sim} w_{i}(K)$ is just the attachment of $L$ and $K$ via the identification of $v_{i}$ with $w_{i}$ for $1 \leqslant i \leqslant n$. In practice the complexes $L$ and $K$ which we use will usually have been defined in $\$ 2$, and as such they contain sets of points with primed or unprimed integer labelings. If the number of, say, unprimed points, $1 \leqslant i \leqslant n$, in $L$ is $n$, and the number of them in $K$ is also $n$, we will abbreviate $i(L) \stackrel{1 \leqslant i \leqslant n}{\sim} i(K)$ by $i(L) \sim i(K)$, it being understood that the identifications are to take place over the full range of $n$ possible values for $i$ in both $L$ and $K$. Similarly, $i(L) \sim i^{\prime}(K)$, $i(L) \sim i^{\prime \prime}(K)$, and $i^{\prime}(L) \sim i^{\prime \prime}(K)$ would be complexes obtained by the indicated identifications over all possible values of $i, i^{\prime}$, or $i^{\prime \prime}$ in $L$ or $K$. The symbol $i(L) \sim(i-1)^{\prime}(K)$ will signify that the point $i$ of $L$ is to be identified with the point $(i-1)^{\prime}$ of $K$ as $i$ runs over its possible values in $L$. We require this notation when the definitions of $L$ and $K$ give a range of $0^{\prime}$ through $n-1^{\prime}$ for the primed points in $K$ and 1 through $n$ for the unprimed ones in $L$. The complex $i\left(C_{3,0}\right) \sim i^{\prime}\left(C_{3,0}\right)$ is illustrated in Figure 3.1(a). For each positive integer $n$ we define the 2-complex $F(n)$ as follows. Referring to the definition of $C_{n, 0}$ given in $\S 2$, we let $V(F(n))=V\left(C_{n, 0}\right)$ $\cup\left\{1^{\prime \prime}, 2^{\prime \prime}, \ldots, n^{\prime \prime}\right\}$, and $F(n)_{(2)}=C_{n, 0(2)} \cup\left\{\left\langle i, i^{\prime}, i^{\prime \prime}\right\rangle / 1 \leqslant i \leqslant n\right\}$. The complex obtained by identifying the points $i^{\prime \prime}, 1 \leqslant i \leqslant n$, of $F(n)$ to a single point $\Omega$ will be denoted by $F^{\prime}(n)$. Note that $\Gamma_{0}(F(n)) \cong \Gamma_{2}(F(n)) \cong \Gamma_{0}\left(F^{\prime}(n)\right) \cong \Gamma_{2}\left(F^{\prime}(n)\right)=\mathbf{Z}_{n}$. We illustrate $F(3)$ and $F^{\prime}(3)$ in Figures 3.1(b) and 3.1(c), where the three points in $F^{\prime}(3)$ labeled 1 are identified. 


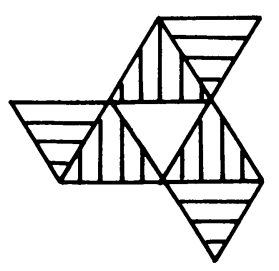

b) $F(3)$

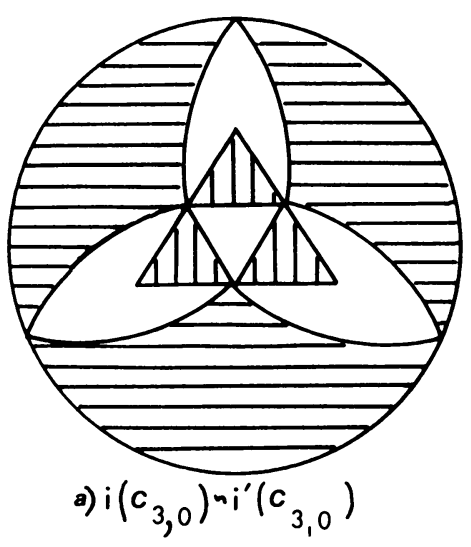

Figure 3.1. Three 2-complexes

In order to state our next result we require some notation. A sum of the form $\sum_{i=r}^{s}\left[x+\delta_{k}(i-r)\right]$ for fixed $x$ and $k>0$ will be denoted by $S_{k}(x, r, s)$. The sum $\sum_{i=r}^{s} x$ will be written $S_{0}(x, r, s)$. Now let $n_{0}=1<n_{1}<n_{2}<\cdots<n_{r}$ be a finite sequence of increasing positive integers, and let $N$ be any positive integer. An expression $S$ of the form

$$
S=S_{k_{1}}\left(x_{1}, 1, n_{1}\right)+S_{k_{2}}\left(x_{2}, n_{1}+1, n_{2}\right)+\cdots+S_{k_{r+1}}\left(x_{r+1}, n_{r}, N\right)
$$

will have its usual meaning when $N>n_{r}$, while if $n_{t-1}+1 \leqslant N \leqslant n_{t}$ for $1 \leqslant t \leqslant r$ then by convention $S$ will be given by

$$
S_{k_{1}}\left(x_{1}, 1, n_{1}\right)+S_{k_{2}}\left(x_{2}, n_{1}+1, n_{2}\right)+\cdots+S_{k_{t}}\left(x_{t}, n_{t-1}+1, N\right) .
$$

Recall that $I\left(p^{\alpha}\right)$ is the number of isomorphism classes among the complexes $C\left(p^{\alpha}, j\right)$ satisfying $\Gamma_{2}\left(C\left(p^{\alpha}, j\right)\right)=\mathbf{Z}_{p^{\alpha}}$, and by Theorem 4 we have $I\left(p^{\alpha}\right)>0$ if and onily if $p^{\alpha} \geqslant 9$.

We may now state the theorem which presents an upper bound for $M_{1}^{(2)}(A)$ when $A$ is an arbitrary finite abelian group. The theorem gives bounds, denoted by $B_{1}^{(2)}\left(\mathbf{Z}_{p^{\alpha}}^{e\left(p^{\alpha}\right)}\right)$, for $M_{1}^{(2)}\left(\mathbf{Z}_{p^{\alpha}}^{e\left(p^{\alpha}\right)}\right)$, from which the bound

$$
M_{1}^{(2)}(A) \leqslant \sum_{p^{\alpha} \mid A} B_{1}^{(2)}\left(\mathbf{Z}_{p^{\alpha}}^{e\left(p^{\alpha}\right)}\right)
$$

follows by construction. The bounds $B_{1}^{(2)}\left(\mathbf{Z}_{p^{\alpha}}^{e\left(p^{\alpha}\right)}\right)$ are given only for those $p^{\alpha}$ satisfying $\alpha>1$ and $p \geqslant 7$, or $\alpha=1$ and $p \geqslant 11$. In the remaining cases the behavior of $B_{1}^{(2)}\left(\mathbf{Z}_{p^{\alpha}}^{e\left(p^{\alpha}\right)}\right)$ is exceptional owing to the smallness of $p^{\alpha}$, and we therefore omit discussion of these cases here. These cases are discussed in detail in [11].

THEOREM 8. Let $A$ be a finite abelian group with canonical factorization $A=\Pi \mathbf{Z}_{p^{\alpha}}^{e\left(p^{\alpha}\right)}$, and for any fixed prime power $p^{\alpha}$ let $n=I\left(p^{\alpha}\right)$. Suppose that for all elementary divisors $p^{\alpha}$ of $A$ we have $\alpha>1$ and $p \geqslant 7$, or $\alpha=1$ and $p \geqslant 11$. Then $M_{1}^{(2)}(A)$ satisfies 
$M_{1}^{(2)}(A) \leqslant \sum_{p^{\alpha} \mid A} B_{1}^{(2)}\left(\mathbf{Z}_{p^{\alpha}}^{e\left(p^{\alpha}\right)}\right)$ where $B_{1}^{(2)}\left(\mathbf{Z}_{p^{\alpha}}^{e\left(p^{\alpha}\right)}\right)$ is given by

$$
\begin{aligned}
& S_{0}\left(p^{\alpha}, 1, n\right)+S_{0}\left(p^{\alpha}+p, n+1, n+\{p / 2\}-3\right) \\
& +S_{0}\left(p^{\alpha}+2 p, n+\{p / 2\}-2, e\left(p^{\alpha}\right)\right) \quad \text { if } p \geqslant 7 \text { and } \alpha>1, \\
& S_{0}(p, 1, n)+S_{0}(2 p, n+1, e(p)) \quad \text { if } p \geqslant 11 \text { and } \alpha=1 .
\end{aligned}
$$

ProOF. The method of proof we employ begins by constructing a 2-cell representation $K\left(\mathbf{Z}_{p^{\alpha}}^{e\left(p^{\alpha}\right)}\right)$ of each factor $\mathbf{Z}_{p^{\alpha}}^{e\left(p^{\alpha}\right)}$ in the decomposition of $A$. We then obtain our bounds $B_{1}^{(2)}\left(\mathbf{Z}_{p^{\alpha}}^{e\left(p^{\alpha}\right)}\right)$ by letting $B_{1}^{(2)}\left(\mathbf{Z}_{p^{\alpha}}^{e\left(p^{\alpha}\right)}\right)=\left|K\left(\mathbf{Z}_{p^{\alpha}}^{e\left(p^{\alpha}\right)}\right)_{(2)}\right|$. The 2-cell representation $K(A)$ of $A$ which we use is $K(A)=\cup_{p^{\alpha} \mid A} K\left(\mathbf{Z}_{p^{\alpha}}^{e\left(p^{\alpha}\right)}\right)$, and it follows that

$$
M_{1}^{(2)}(A) \leqslant\left|K(A)_{(2)}\right|=\sum_{p^{\alpha} \mid A} B_{1}^{(2)}\left(\mathbf{Z}_{p^{\alpha}}^{e\left(p^{\alpha}\right)}\right) .
$$

In what follows, then, we construct a 2-cell representation $K\left(\mathbf{Z}_{p^{\alpha}}^{e\left(p^{\alpha}\right)}\right)$ for each subgroup $\mathbf{Z}_{p^{\alpha}}^{e\left(p^{\alpha}\right)}$ appearing in the factorization of $A$ such that the value of

$$
\left|K\left(\mathbf{Z}_{p^{\alpha}}^{e\left(p^{\alpha}\right)}\right)_{(2)}\right|=B_{1}^{(2)}\left(\mathbf{Z}_{p^{\alpha}}^{e\left(p^{\alpha}\right)}\right)
$$

is as claimed.

Suppose first then that $\alpha>1$ and $p \geqslant 7$. We will require three types of 2-complexes in our construction. The first type will be the $p^{\alpha}$-cyclic complex $C\left(p^{\alpha}, j\right)$. By Theorem 4 there exist $I\left(p^{\alpha}\right)>0$ mutually nonisomorphic complexes of the form $C\left(p^{\alpha}, j\right)$, each satisfying $\Gamma_{2}\left(C\left(p^{\alpha}, j\right)\right)=\mathbf{Z}_{p^{\alpha}}$. Now define $H_{1}$ to be some $C\left(p^{\alpha}, j_{0}\right)$ satisfying $\Gamma_{2}\left(C\left(p^{\alpha}, j_{0}\right)\right)=\mathbf{Z}_{p^{\alpha}}$, and for each $i, 1 \leqslant i \leqslant I\left(p^{\alpha}\right)$, let $H_{i}$ be a complex $C\left(p^{\alpha}, j\right)$ satisfying $\Gamma_{2}\left(C\left(p^{\alpha}, j\right)\right)=\mathbf{Z}_{p^{\alpha}}$ such that $H_{i} \neq H_{r}$ for $r<i$.

We now define the second type of complex. It is shown that each example of this type has cell group $\mathbf{Z}_{p^{\alpha}}$, and the maximum number of mutually nonisomorphic examples is determined. Define the complex $Y\left(j, p^{\alpha}\right)$ by $Y\left(j, p^{\alpha}\right)=i(C(p, j)) \sim$ $(i-1)^{\prime}\left({ }_{p} C_{p^{\alpha}}\right)$, where $j$ satisfies $4 \leqslant j \leqslant\{p / 2\}$. As abbreviations, we write $Y$ for $Y\left(j, p^{\alpha}\right)$ when $j$ and $p^{\alpha}$ are fixed, and $Y(j)$ for $Y\left(j, p^{\alpha}\right)$ when only $p^{\alpha}$ is fixed.

We now claim that $\Gamma_{2}(Y)=\mathbf{Z}_{p^{\alpha}}$. To see this, denote the subcomplex ${ }_{p} C_{p^{\alpha}}$ of $Y$ by $C$ for brevity, and observe there is a natural homomorphism $f: \Gamma_{2}(Y) \rightarrow \Gamma_{2}(C)$ given by restriction to $C$, i.e., $f(\alpha)=\left.\alpha\right|_{C}$. The map $f$ is injective since if $\left.\sigma\right|_{C}=1$, then $\sigma=1$ on all of $Y_{(2)}$. It follows that $\Gamma_{2}(Y)$ is a subgroup of $\Gamma_{2}(C)=D_{2 p^{\alpha}}$, the dihedral group of order $2 p^{\alpha}$. It remains to show that $\Gamma_{2}(Y)$ contains no involution $\tau$. Now $\Gamma_{2}(Y)$ is clearly invariant on $C$, and hence such a $\tau$ when restricted to $C$ may be assumed without loss of generality to act on points according to $\tau(j)=p^{\alpha}+3-j$ and $\tau\left(j^{\prime}\right)=(p+3-j)^{\prime}$. But then $\tau$ does not preserve the cells of the subcomplex $C(p, j)$ since $4 \leqslant j \leqslant\{p / 2\}$. Thus $\Gamma_{2}(Y)$ contains no such $\tau$, and hence $\Gamma_{2}(Y)=\mathbf{Z}_{p^{\alpha}}$ as claimed. To summarize, the complex ${ }_{p} C_{p^{\alpha}}$ has dihedral symmetry, but attachment of $C(p, j)$ via the identifications $i \sim(i-1)^{\prime}, 1 \leqslant i \leqslant p$, destroys all the involutions. The cell group of the resulting complex is then just $\mathbf{Z}_{p^{\alpha}}$.

Next we assert that $Y(j) \not Y(k)$ if $j \neq k$. Suppose the contrary, and let $f$ : $Y(j) \rightarrow Y(k)$ be an isomorphism. Let $C_{1}$ and $C_{2}$ be copies of ${ }_{p} C_{p^{\alpha}}$ in $Y(j)$ and $Y(k)$ respectively. Clearly $f\left(C_{1}\right)=C_{2}$, and hence $f$ may be viewed as an element of 
$\Gamma_{0}\left({ }_{p} C_{p^{\alpha}}\right)=D_{2 p^{\alpha}}$. Therefore we have $f=\sigma^{x} \tau^{y}$, where $0 \leqslant x \leqslant p^{\alpha}-1,0 \leqslant y \leqslant 1$, $\sigma=\left(12 \cdots p^{\alpha}\right)\left(1^{\prime} 2^{\prime} \cdots p^{\prime}\right)$, and

$$
\tau=(\{p / 2\}+1)\left(\{p / 2\}+1^{\prime}\right) \prod_{j=1}^{\left\{p^{\alpha} / 2\right\}}\left(j, p^{\alpha}+3-j\right) \prod_{j=1}^{\{p / 2\}}\left(j^{\prime}, p+3-j^{\prime}\right) .
$$

Clearly we have $\sigma^{x}(Y(j))=Y(j)$ and $\tau(Y(j))=Y(p+3-j)$. Hence we get $k=j$ or $k=p+3-j$, both of which are disallowed by the conditions $k \neq j$ and $k, j \leqslant\{p / 2\}$. It follows that $Y(j) \neq Y(k)$ as claimed. Finally, note that $\left|Y(j)_{(2)}\right|=$ $p^{\alpha}+p$ for all $j$.

As the third type of complex, we will define for each positive integer $k$ a 2-complex $T_{k}$ satisfying $\Gamma_{2}\left(T_{k}\right) \cong \mathbf{Z}_{p^{\alpha}}^{k}$. For later use, the $p$ points of $Y(j)$ in ${ }_{p} C_{p^{\alpha}} \cap C(p, j)$ will retain the names $i^{\prime}, 0 \leqslant i \leqslant p-1$, they had in ${ }_{p} C_{p^{\alpha}}$. Define $T_{1}$ by letting

$$
\begin{aligned}
T_{1} & =i\left(B_{p}\right) \sim(i-1)^{\prime}(Y(j)) \\
& =i\left(B_{p}\right) \sim(i-1)^{\prime}\left[i(C(p, j)) \sim(i-1)^{\prime}\left({ }_{p} C_{p^{\alpha}}\right)\right]
\end{aligned}
$$

for some $j$. Thus $T_{1}$ is formed by identifying the nonspinal points of a $p$-book in a one-one manner with the points $i^{\prime}, 0 \leqslant i \leqslant p-1$, of $Y$. The proof that $\Gamma_{2}\left(T_{1}\right)=\mathbf{Z}_{p^{\alpha}}$ is almost identical to the proof of $\Gamma_{2}(Y(j)) \cong \mathbf{Z}_{p^{\alpha}}$, and is hence omitted. Let $s$ be a spinal point on the $p$-book $T_{1}$, and define $T_{2}$ by

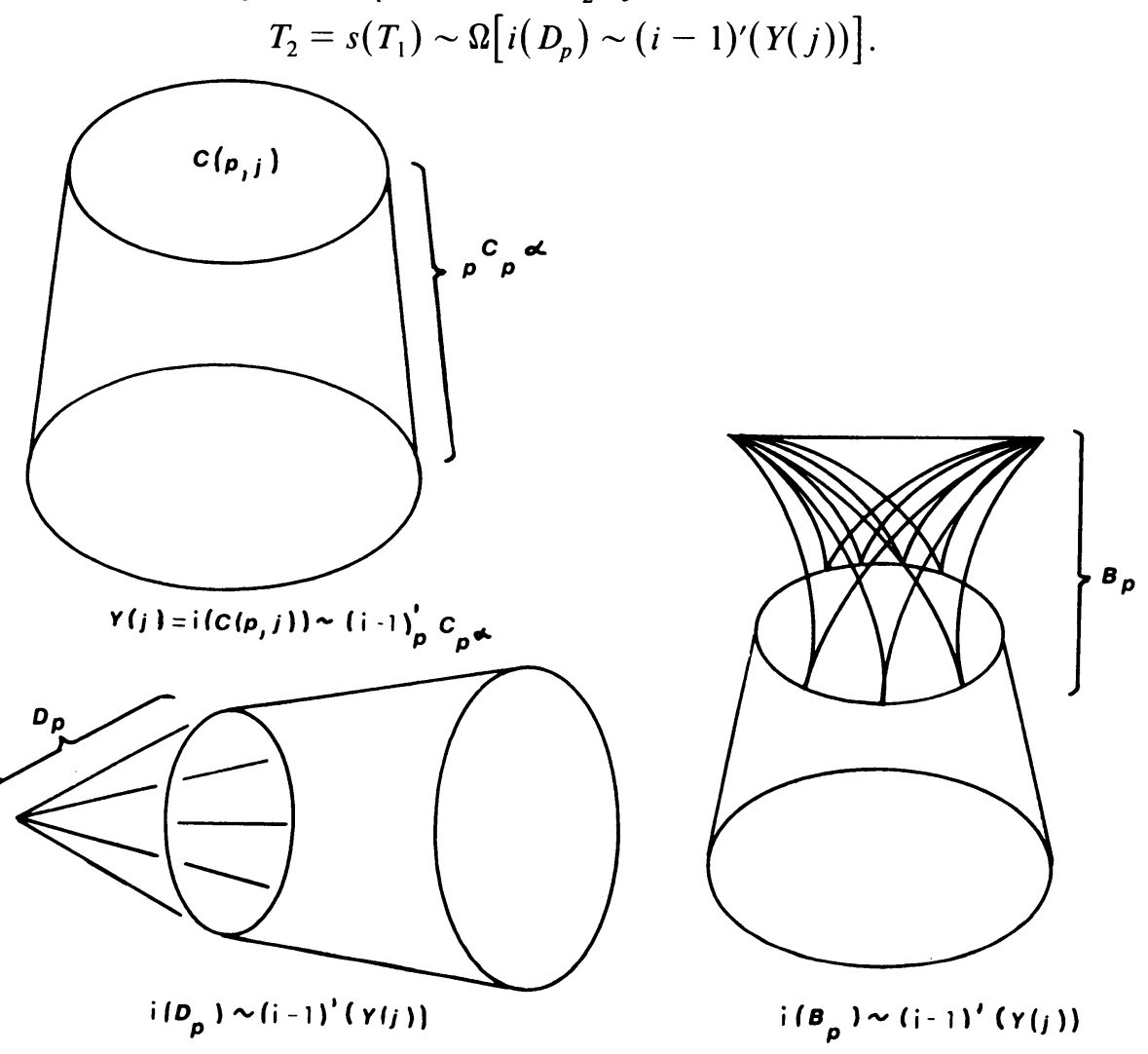

FIGURE 3.2. Complexes used in constructing $T_{k}$ 


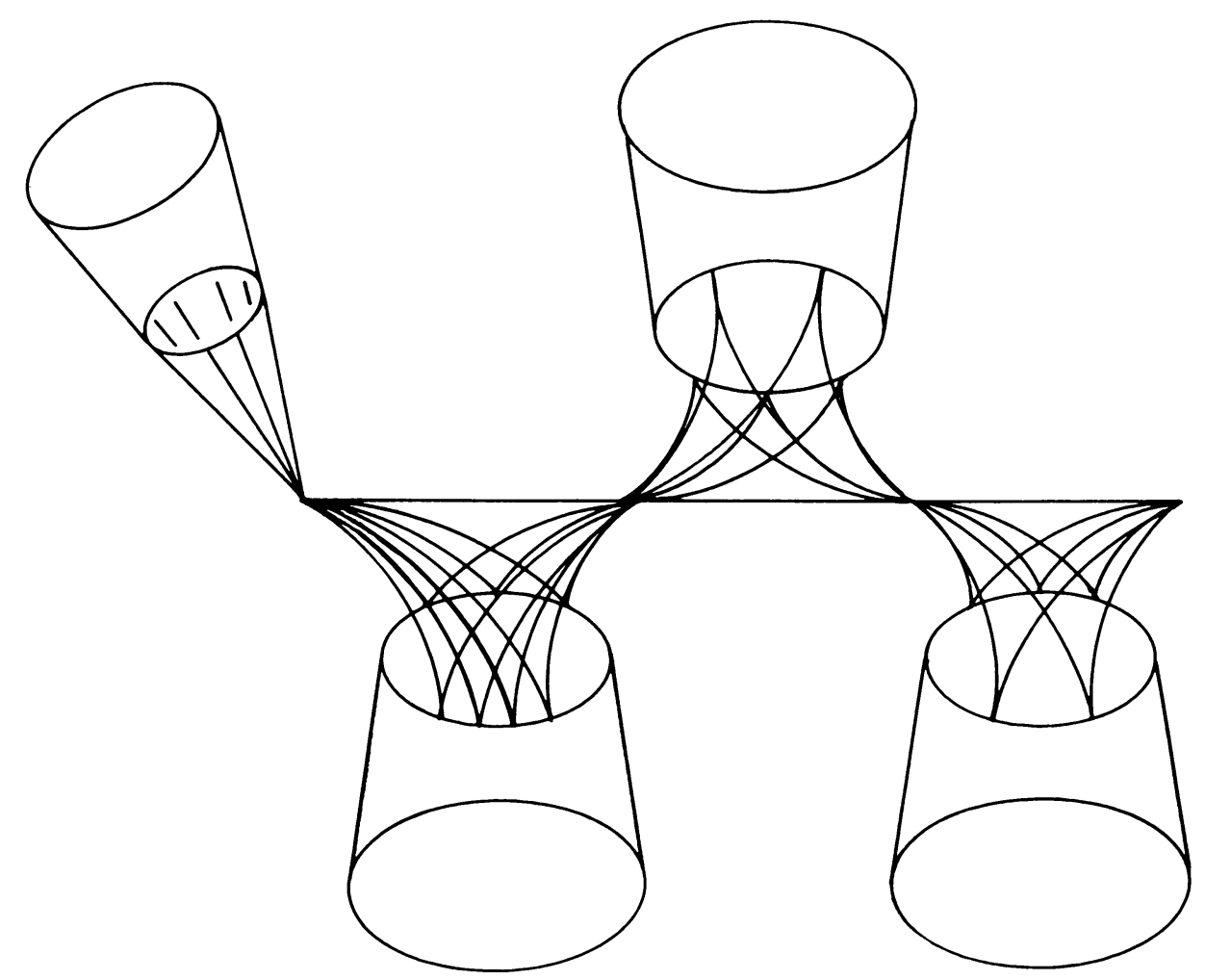

Figure 3.3. The complex $T_{4}$

Clearly we have $\Gamma_{2}\left(i\left(D_{p}\right) \sim(i-1)^{\prime}(Y(j))\right) \cong \mathbf{Z}_{p^{\alpha}}$, and hence $\Gamma_{2}\left(T_{2}\right)=\Gamma_{2}\left(T_{1}\right) \times \mathbf{Z}_{p^{\alpha}}$ $\cong \mathbf{Z}_{p^{\alpha}}^{2}$. Having constructed $T_{k-1}$, we define $T_{k}$ recursively by letting $T_{k}=s\left(T_{k-1}\right) \sim$ $t\left(T_{1}\right)$ where $s$ and $t$ are spinal points of $p$-books in $T_{k-1}$ and $T_{1}$ respectively such that $s \neq \Omega$ if $k=3$ and $s \notin T_{k-2}$ if $k \geqslant 4$. The "building blocks" of $T_{k}$ are illustrated in Figure 3.2 and $T_{4}$ is shown in Figure 3.3.

We now show that $\Gamma_{2}\left(T_{k}\right) \cong \mathbf{Z}_{p^{\alpha}}^{k}$. It may be assumed that $k \geqslant 2$ as the case $k=1$ has already been treated. Define the subcomplex $F_{j}, 1 \leqslant j \leqslant k$, of $T_{k}$ to be

$$
F_{j}=\left\langle T_{j(2)} \backslash T_{j-1(2)}\right\rangle \text {. }
$$

Thus $F_{j}=T_{1}$ for $j \neq 2$ and $F_{2}=i\left(D_{p}\right) \sim(i-1)^{\prime}(Y(t))$ for some $t$, so that $F_{j}$ may be informally described as the $j$ th "floor" of the "tower" $T_{k}$. We know that $\Gamma_{2}\left(F_{j}\right) \cong \mathbf{Z}_{p^{\alpha}}$ for all $j$. Our object is to show that there exists an isomorphism

$$
f: \prod_{j=1}^{k} \Gamma_{2}\left(F_{j}\right) \rightarrow \Gamma_{2}\left(T_{k}\right),
$$

and the statement $\Gamma_{2}\left(T_{k}\right) \cong \mathbf{Z}_{p^{\alpha}}^{k}$ would follow. Let $\left(\sigma_{1}, \sigma_{2}, \ldots, \sigma_{k}\right)$ by any $k$-tuple satisfying $\sigma_{i} \in \Gamma_{2}\left(F_{i}\right), 1 \leqslant i \leqslant k$. To each such $k$-tuple there corresponds an element $\left(\sigma_{1}, \sigma_{2}, \ldots, \sigma_{k}\right)^{e}$ of $\Gamma_{2}\left(T_{k}\right)$ given by $\left.\left(\sigma_{1}, \sigma_{2}, \ldots, \sigma_{k}\right)^{e}\right|_{F_{j}}=\sigma_{j}$. We then define the homomorphism $f: \prod_{j=1}^{k} \Gamma_{2}\left(F_{j}\right) \rightarrow \Gamma_{2}\left(T_{k}\right)$ by $f\left(\sigma_{1}, \sigma_{2}, \ldots, \sigma_{k}\right)=\left(\sigma_{1}, \sigma_{2}, \ldots, \sigma_{k}\right)^{e}$. Observe that $f$ is injective, since $\left(\sigma_{1}, \sigma_{2}, \ldots, \sigma_{k}\right)^{e}=1$ in $\Gamma_{2}\left(T_{k}\right)$ implies that $\sigma_{j}=1$ for all 
$j$. For surjectivity, observe that a typical element $\tau$ of $\Gamma_{2}\left(T_{k}\right) \backslash f\left(\Pi_{j=1}^{k} \Gamma_{2}\left(F_{j}\right)\right)$ must fail to be invariant on some $F_{j}$. Since the $F_{j}$ 's are preserved under $\Gamma_{2}\left(T_{k}\right)$, it follows that $\tau$ would satisfy $\tau\left(F_{j}\right)=F_{l}$ for some pair $j, l, j \neq l$. But this action on $T_{k}$ has been disallowed because $T_{k}$ has been "rooted" at one end with the complex $i\left(D_{p}\right) \sim(i-1)^{\prime}(Y(t))$. Hence no such $\tau$ exists and $f$ is thus surjective. It follows that $f: \Pi_{j=1}^{k} \Gamma_{2}\left(F_{j}\right) \rightarrow \Gamma_{2}\left(T_{k}\right)$ is an isomorphism, and thus $\Gamma_{2}\left(T_{k}\right) \cong \mathbf{Z}_{p^{\alpha}}^{k}$ as required.

We may now describe the 2-cell representation $K\left(\mathbf{Z}_{p^{2}}^{e\left(p^{\alpha}\right)}\right)$ of $\mathbf{Z}_{p^{2}}^{e\left(p^{\alpha}\right)}$. Define $H_{I\left(p^{\alpha}\right)+1}$ to be $Y(j)$ for some $j$, and then for each integer $r$ satisfying $I\left(p^{\alpha}\right)+2 \leqslant r$ $\leqslant I\left(p^{\alpha}\right)+\{p / 2\}-3$, let $H_{r} \cong Y(k)$ in such a way that $H_{r} \neq H_{s}$ for $s<r$. We observe that such an assignment is possible since it was shown above that the $\{p / 2\}-3$ complexes $Y(j), 4 \leqslant j \leqslant\{p / 2\}$, are mutually nonisomorphic. Now define $K\left(\mathbf{Z}_{p^{\alpha}}^{e\left(p^{\alpha}\right)}\right)$ by

$$
K\left(\mathbf{Z}_{p^{e}}^{e\left(p^{\alpha}\right)}\right)=\left\{\begin{array}{cc}
\bigcup_{i=1}^{I\left(p^{\alpha}\right)+\{p / 2\}-3} H_{i} \cup T_{e\left(p^{\alpha}\right)-\left(I\left(p^{\alpha}\right)+\{p / 2\}-3\right),} & \text { if } e\left(p^{\alpha}\right)>I\left(p^{\alpha}\right)+\{p / 2\}-3, \\
\bigcup_{i=1}^{e\left(p^{\alpha}\right)} H_{i} & \text { if } e\left(p^{\alpha}\right) \leqslant I\left(p^{\alpha}\right)+\{p / 2\}-3 .
\end{array}\right.
$$

We now claim that

$$
\Gamma_{2}\left(K\left(\mathbf{Z}_{p^{\alpha}}^{e\left(p^{\alpha}\right)}\right)\right)=\mathbf{Z}_{p^{\alpha}}^{e\left(p^{\alpha}\right)} .
$$

For convenience write $K^{\prime}$ for the complex

$$
\bigcup_{i=1}^{I\left(p^{\alpha}\right)+\{p / 2\}-3} H_{i}
$$

Now $K^{\prime}$ has $I\left(p^{\alpha}\right)+\{p / 2\}-3$ connected components, and we have shown that each has cell group $\mathbf{Z}_{p^{\alpha}}$ while no two of these components are isomorphic. It follows that $\Gamma_{2}\left(K^{\prime}\right)=\mathbf{Z}_{p^{\alpha}}^{I\left(p^{\alpha}\right)+\{p / 2\}-3}$. Clearly $T_{e\left(p^{\alpha}\right)-\left(I\left(p^{\alpha}\right)+\{p / 2\}-3\right)}$ is connected, and is isomorphic to no connected component of $K^{\prime}$. We thus get

$$
\Gamma_{2}\left(K\left(\mathbf{Z}_{p^{\alpha}}^{e\left(p^{\alpha}\right)}\right)\right)=\Gamma_{2}\left(K^{\prime}\right) \times \Gamma_{2}\left(T_{e\left(p^{\alpha}\right)-\left(I\left(p^{\alpha}\right)+\{p / 2\}-3\right)}\right)=\mathbf{Z}_{p^{\alpha}}^{e\left(p^{\alpha}\right)} .
$$

Hence our upper bound for $M_{1}^{(2)}\left(\mathbf{Z}_{p^{\alpha}}^{e\left(p^{\alpha}\right)}\right)$ when $p \geqslant 7$ and $\alpha>1$ is

$$
\begin{aligned}
M_{1}^{(2)}\left(\mathbf{Z}_{p^{\alpha}}^{e\left(\alpha^{\alpha}\right)}\right) \leqslant & S_{0}\left(p^{\alpha}, 1, n\right)+S_{0}\left(p^{\alpha}+p, n+1, n+\{p / 2\}-3\right) \\
& +S_{0}\left(p^{\alpha}+2 p, n+\{p / 2\}-2, e\left(p^{\alpha}\right)\right) .
\end{aligned}
$$

We now consider the case $\alpha=1$ and $p \geqslant 11$. Since $I(p)>0$, we may define $H_{i}$, $1 \leqslant i \leqslant I(p)$, as a full set of $I(p)$ representatives, one from each isomorphism class, of the set of complexes $C(p, j)$ satisfying $\Gamma_{2}(C(p, j)) \cong \mathbf{Z}_{p}$. Next we define a complex $T_{k}^{\prime}$ for each integer $k>0$ similar to the complex $T_{k}$ used in forming $K\left(\mathbf{Z}_{p^{\alpha}}^{e\left(p^{\alpha}\right)}\right)$ for $p \geqslant 7$ and $\alpha>1$. Let $T_{1}^{\prime}=i\left(D_{p}\right) \sim i(C(p, t))$ for some $C(p, t)$ satisfying $\Gamma_{2}(C(p, t)) \cong \mathbf{Z}_{p}$, and we observe that $\Gamma_{2}\left(T_{1}^{\prime}\right) \cong \mathbf{Z}_{p}$. Now let $u$ be a spinal 
point on the $p$-book of $i\left(B_{p}\right) \sim i(C(p, t))$, and define $T_{2}^{\prime}$ to be

$$
T_{2}^{\prime}=\Omega\left(T_{1}^{\prime}\right) \sim u\left[i\left(B_{p}\right) \sim i(C(p, t))\right] .
$$

For $k \geqslant 3$ we define $T_{k}^{\prime}$ recursively. Let $s$ be the spinal point on the $p$-book of a subcomplex $i\left(B_{p}\right) \sim i(C(p, t))$ of $T_{k-1}^{\prime}$ such that $s \notin T_{k-2}^{\prime}$. We then let $T_{k}^{\prime}=$ $s\left(T_{k-1}^{\prime}\right) \sim u\left[i\left(B_{p}\right) \sim i(C(p, t))\right]$. The proof that $\Gamma_{2}\left(T_{k}\right) \cong \mathbf{Z}_{p^{\alpha}}^{k}$ can be used almost verbatim to show that $\Gamma_{2}\left(T_{k}^{\prime}\right)=\mathbf{Z}_{p}^{k}$. Now define $K\left(\mathbf{Z}_{p}^{e(p)}\right)$ by

$$
K\left(\mathbf{Z}_{p}^{e(p)}\right)= \begin{cases}\bigcup_{i=1}^{I(p)} H_{i} \cup T_{e(p)-I(p)}^{\prime} & \text { if } e(p)>I(p), \\ \bigcup_{i=1}^{e(p)} H_{i} & \text { if } e(p) \leqslant I(p) .\end{cases}
$$

The resulting bound for $M_{1}^{(2)}\left(\mathbf{Z}_{p}^{e(p)}\right), p \geqslant 11$, is

$$
M_{1}^{(2)}\left(\mathbf{Z}_{p}^{e(p)}\right) \leqslant\left|K\left(\mathbf{Z}_{p}^{e(p)}\right)_{(2)}\right|=S_{0}(p, 1, n)+S_{0}(2 p, n+1, e(p)) .
$$

We omit here the construction of $K\left(\mathbf{Z}_{p^{\alpha}}^{e\left(p^{\alpha}\right)}\right)$ in all remaining cases for $p^{\alpha}$ as these involve "ad hoc" features necessitated by the smallness of $p^{\alpha}$. These include the use of the complexes $F(n)$ and $F^{\prime}(n)$ "mounted" on special complexes having identity automorphism group. A complete description of these constructions is available in [11].

We now discuss the construction of minimal pure $n$-dimensional simplicial complexes $K$ having given $A \cong \Gamma_{n}(K)$ for $n \geqslant 3$ or given $A \cong \Gamma_{0}(K)$ for $n \geqslant 2$. Our object is to find upper bounds for $M_{1}^{(n)}(A)$ and $M_{0}^{(n)}(A)$ in these dimensions $n$. It will be seen that these bounds give the exact value of $M_{1}^{(n)}(A)$ when $n \geqslant 4$ and of $M_{0}^{(n)}(A)$ when $n=2$ for "almost all" $A$. The $n$-complexes at which the values of $M_{1}^{(n)}(A)$ are realized arise as analogues of the 2-complexes $T_{k}$ described in the proof of Theorem 8. In addition we will make use of the topological cone over the 2-complexes $C\left(p^{\alpha}, j\right)$ discussed in $\S 2$.

Let $K$ be a pure $n$-complex, and recall that $K_{(n)}$ is its set of maximal simplexes. We describe a method of constructing complexes of dimension greater than $n$ containing $K$ as a subcomplex. The $(n+r)$-cone over $K$, written $C_{n+r}(K)$, is the pure $(n+r)$ dimensional simplicial complex defined by $V\left(C_{n+r}(K)\right)=V(K) \cup\left\{v_{1}, v_{2}, \ldots, v_{r}\right\}$, $C_{n+r}(K)_{(n+r)}=\left\{\left\langle\alpha, v_{1}, v_{2}, \ldots, v_{r}\right\rangle / \alpha \in K_{(n)}\right\}$. The 3 -cone over $2 P K_{3}, C_{3}\left(2 P K_{3}\right)$, is illustrated in Figure 3.4.
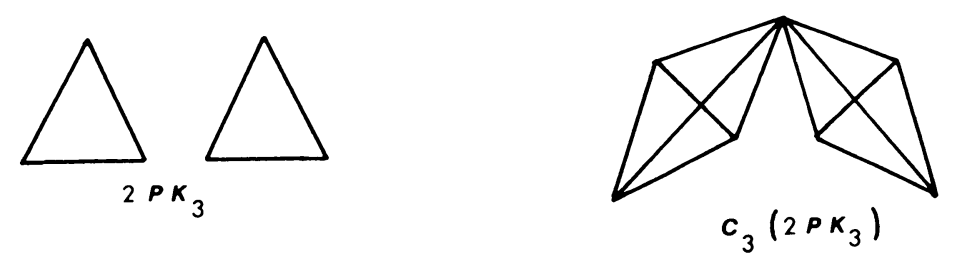

FIgURE 3.4. The complexes $2 P K_{3}$ and $C_{3}\left(2 P K_{3}\right)$ 
We define $P_{n+r}(K)$ to be the set of points $\left\{v_{1}, v_{2}, \ldots, v_{r}\right\}$ in $C_{n+r}(K)$, and the elements of $P_{n+r}(K)$ will be called perspectivity points or perspectivities of $C_{n+r}(K)$.

We are now ready for Theorem 9 which gives upper bounds for $M_{1}^{(n)}(A)$ in dimension $n \geqslant 3$. In order to avoid small exceptional cases we will assume that all elementary divisors $p^{\alpha}$ of $A$ satisfy $p^{\alpha} \geqslant 9$.

THEOREM 9. Let $A$ be a finite abelian group, and let $A=\prod \mathbf{Z}_{p^{\alpha}}^{e\left(p^{\alpha}\right)}$ be the canonical factorization of $A$. For fixed $p^{\alpha} \geqslant 9$, let $\gamma=I\left(p^{\alpha}\right)$ (determined in Theorem 4) be the number of isomorphism classes of $C\left(p^{\alpha}, j\right)$ satisfying $\Gamma_{2}\left(C\left(p^{\alpha}, j\right)\right)=\mathbf{Z}_{p^{\alpha}}$. Suppose that $p^{\alpha} \geqslant 9$ for all $p^{\alpha}$ dividing $A$. Then $M_{1}^{(n)}(A)$ satisfies

$$
M_{\mathrm{l}}^{(n)}(A) \leqslant \sum_{p^{\alpha} \mid A} B_{1}^{(n)}\left(\mathbf{Z}_{p^{\alpha}}^{e\left(p^{\alpha}\right)}\right)
$$

where $B_{1}^{(n)}\left(\mathbf{Z}_{p^{\alpha}}^{e\left(p^{\alpha}\right)}\right)$ is given for $n \geqslant 4$ by

(1) $p^{\alpha} e\left(p^{\alpha}\right)$ if $p^{\alpha} \geqslant 17$

(2) $S_{0}\left(p^{\alpha}, 1,1\right)+S_{0}\left(p^{\alpha}, 2, e\left(p^{\alpha}\right)\right)+1$ if $9 \leqslant p^{\alpha} \leqslant 13$, and for $n=3$ by

(3)

$$
\begin{aligned}
S_{0}\left(p^{\alpha}, 1, \gamma 2^{\gamma-1}\right) & +S_{4 \gamma-2}\left(p^{\alpha}, 1+\gamma 2^{\gamma-1}, \gamma\left(2^{\gamma-1}+4\right)-3\right) \\
& +S_{3 \gamma-1}\left(p^{\alpha}, \gamma\left(2^{\gamma-1}+4\right)-2, e\left(p^{\alpha}\right)\right)
\end{aligned}
$$

if $p^{\alpha} \geqslant 23$,

(4) $S_{0}\left(p^{\alpha}, 1,4\right)+S_{5}\left(p^{\alpha}, 5,8\right)+S_{5}\left(p^{\alpha}, 9, e\left(p^{\alpha}\right)\right)$ if $p^{\alpha}=17$ or 19 ,

(5) $S_{0}\left(p^{\alpha}, 1,1\right)+\left(1+p^{\alpha}\right)\left(e\left(p^{\alpha}\right)-1\right)$ if $9 \leqslant p^{\alpha} \leqslant 13$.

Proof. Again we proceed by first constructing an $n$-cell representation $K^{(n)}\left(\mathbf{Z}_{p^{\alpha}}^{e\left(p^{\alpha}\right)}\right)$ of each direct factor $\mathbf{Z}_{p^{\alpha}}^{e\left(p^{\alpha}\right)}$ of $A$. We then obtain a representation $K^{(n)}(A)$ of $A$ by using the disjoint union

$$
K^{(n)}(A)=\bigcup_{p^{\alpha} \mid A} K^{(n)}\left(\mathbf{Z}_{p^{\alpha}}^{e\left(p^{\alpha}\right)}\right) .
$$

Letting $B_{1}^{(n)}\left(\mathbf{Z}_{p^{\alpha}}^{e\left(p^{\alpha}\right)}\right)$ be the number of $n$-cells in $K^{(n)}\left(\mathbf{Z}_{p^{\alpha}}^{e\left(p^{\alpha}\right)}\right)$, we get

$$
M_{1}^{(n)}(A) \leqslant\left|K^{(n)}(A)_{(n)}\right|=\sum_{p^{\alpha} \mid A} B_{1}^{(n)}\left(\mathbf{Z}_{p^{\alpha}}^{e\left(p^{\alpha}\right)}\right) .
$$

It therefore remains to construct $K^{(n)}\left(\mathbf{Z}_{p^{\alpha}}^{e\left(p^{\alpha}\right)}\right)$ for $p^{\alpha} \mid A$, to show that $B_{1}^{(n)}\left(\mathbf{Z}_{p^{e}}^{e\left(p^{\alpha}\right)}\right)$ has the required value, and to observe that $\Gamma_{n}\left(K^{(n)}(A)\right) \cong A$. We describe the constructions only for $n \geqslant 4$ to avoid unimportant complications. The constructions for $n=3$ are detailed in [11].

Suppose first that $p^{\alpha} \geqslant 17$. We construct an $n$-dimensional analogue $T_{k}^{(n)}$ of the tower $T_{k}$ used in the case $n=2$. By Theorem 4 there exists integers $j, k$ satisfying $\Gamma_{2}\left(C\left(p^{\alpha}, j\right)\right) \cong \Gamma_{2}\left(C\left(p^{\alpha}, k\right)\right) \cong \mathbf{Z}_{p^{\alpha}}$ and $C\left(p^{\alpha}, j\right) \neq C\left(p^{\alpha}, k\right)$. Define $T_{1}^{(n)}$ and $T_{2}^{(n)}$ by $C_{n}\left(C\left(p^{\alpha}, j\right)\right)$ and $s\left(T_{1}^{(n)}\right) \sim t\left(C_{n}\left(C\left(p^{\alpha}, k\right)\right)\right)$, where $s \in P_{n}\left(C\left(p^{\alpha}, j\right)\right)$ and $t \in$ $P_{n}\left(C\left(p^{\alpha}, k\right)\right)$. Now suppose that we have constructed $T_{k}^{(n)}$ for $1 \leqslant k \leqslant N-1$. We then define $T_{N}^{(n)}$ by $T_{N}^{(n)}=s\left(T_{N-1}^{(n)}\right) \sim t\left(C_{n}\left(C\left(p^{\alpha}, k\right)\right)\right)$, where $s \in P_{n}\left(C\left(p^{\alpha}, k\right)\right)$, 
$s \notin T_{N-2}$, and $t \in P_{n}\left(C\left(p^{\alpha}, k\right)\right)$. Thus $s \in T_{N-1}^{(n)}$ is a perspectivity point of the copy of $C_{n}\left(C\left(p^{\alpha}, k\right)\right)$ not contained in $T_{N-2}^{(n)}$, and $T_{N}^{(n)}$ is obtained by attaching $C_{n}\left(C\left(p^{\alpha}, k\right)\right)$ to $T_{N-1}^{(n)}$ via the identification of $s$ and $t$. The complex $T_{N}^{(n)}$ may be viewed as a "tower" having $N$ "floors" $F_{i}, 1 \leqslant i \leqslant N$, with $F_{1} \cong C_{n}\left(C\left(p^{\alpha}, j\right)\right)$, $F_{i} \cong C_{n}\left(C\left(p^{\alpha}, k\right)\right)$ for $2 \leqslant i \leqslant N$, and

$$
F_{i}=\left\langle T_{i(n)}^{(n)} \backslash T_{i-1(n)}^{(n)}\right\rangle .
$$

Each floor is attached to the ones immediately "above" or "below" it by the identification of perspectivities. An illustration of $T_{l}^{(n)}, l \geqslant 2$, is given in Figure 3.5, perspectivity points being dotted.
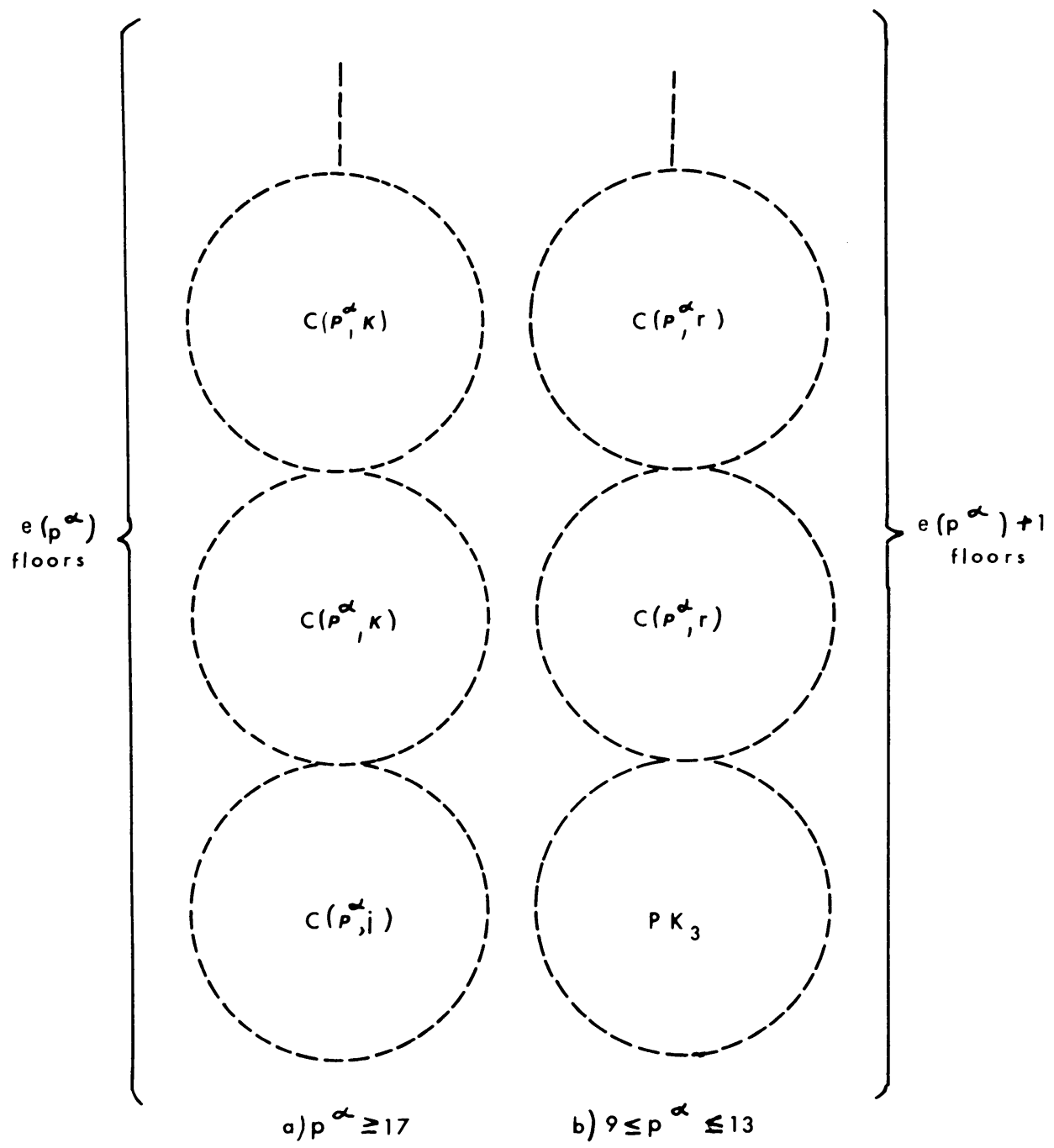

FIGURE 3.5. The complex $K^{(n)}\left(\mathbf{Z}_{p^{\alpha}}^{e\left(p^{\alpha}\right)}\right)$ for $p^{\alpha} \geqslant 9$ and $n \geqslant 4$ 
We now claim that $\Gamma_{n}\left(T_{l}^{(n)}\right) \cong \mathbf{Z}_{p^{\alpha}}^{l}$. Since $\Gamma_{n}\left(F_{i}\right) \cong \mathbf{Z}_{p^{\alpha}}$ for all $i$, it suffices to give an isomorphism $f: \Pi_{i=1}^{l} \Gamma_{n}\left(F_{i}\right) \rightarrow \Gamma_{n}\left(T_{l}^{(n)}\right)$. For any $l$-tuple $\left(\sigma_{1}, \sigma_{2}, \ldots, \sigma_{l}\right)$ with $\sigma_{i} \in$ $\Gamma_{n}\left(F_{i}\right)$, define $f\left(\sigma_{1}, \sigma_{2}, \ldots, \sigma_{l}\right) \in \Gamma_{n}\left(T_{l}^{(n)}\right)$ by $\left.f\left(\sigma_{1}, \sigma_{2}, \ldots, \sigma_{l}\right)\right|_{F_{i}}=\sigma_{i}$ for $1 \leqslant i \leqslant l$. The injectivity of $f$ is immediate from its definition. The surjectivity follows from the fact that $T_{l}^{(n)}$ is rooted at $F_{1}$ and the consequence that any element of $\Gamma_{n}\left(T_{l}^{(n)}\right)$ is invariant on each $F_{i}$. We may therefore define $K^{(n)}\left(\mathbf{Z}_{p^{\alpha}}^{e\left(p^{\alpha}\right)}\right)$ by $K^{(n)}\left(\mathbf{Z}_{p^{\alpha}}^{e\left(p^{\alpha}\right)}\right)=T_{e\left(p^{\alpha}\right)}^{(n)}$. The resulting upper bound for $p^{\alpha} \geqslant 17$ is $M_{1}^{(n)}\left(\mathbf{Z}_{p^{\alpha}}^{e\left(p^{\alpha}\right)}\right) \leqslant p^{\alpha} e\left(p^{\alpha}\right)$.

Suppose now that $9 \leqslant p^{\alpha} \leqslant 13$. We will modify slightly the construction given above. That construction cannot be carried out here since Theorem 4 yields only one integer $r$ satisfying $\Gamma_{2}\left(C\left(p^{\alpha}, r\right)\right) \cong \mathbf{Z}_{p}$ when $9 \leqslant p^{\alpha} \leqslant 13$. Instead we let $T_{1}^{(n)}=$ $C_{n}\left(C\left(p^{\alpha}, r\right)\right)$ and

$$
T_{2}^{(n)}=t\left[u\left(T_{1}^{(n)}\right) \sim v\left(C_{n}\left(P K_{3}\right)\right)\right] \sim s\left(C_{n}\left(C\left(p^{\alpha}, r\right)\right)\right),
$$

where $t, u \in P_{n}\left(C\left(p^{\alpha}, r\right)\right) \subset V\left(T_{1}^{(n)}\right), t \neq u, v \in P_{n}\left(P K_{3}\right)$, and $s \in P_{n}\left(C\left(p^{\alpha}, r\right)\right)$. Having constructed $T_{q}^{(n)}$ for $1 \leqslant q \leqslant N-1$, define $T_{N}^{(n)}$ by

$$
T_{N}^{(n)}=s\left(T_{N-1}^{(n)}\right) \sim t\left(C_{n}\left(C\left(p^{\alpha}, r\right)\right)\right),
$$

where $s \in P_{n}\left(C\left(p^{\alpha}, r\right)\right), s \notin T_{N-2}^{(n)}$, and $t \in P_{n}\left(C\left(p^{\alpha}, r\right)\right)$. The complex $T_{e\left(p^{\alpha}\right)}^{(n)}$ in this case is illustrated in Figure 3.5(b). We now define $K^{(n)}\left(\mathbf{Z}_{p^{\alpha}}^{e\left(p^{\alpha}\right)}\right)$ as $T_{e\left(p^{\alpha}\right)}^{(n)}$. The proof that $\Gamma_{n}\left(K^{(n)}\left(\mathbf{Z}_{p^{\alpha}}^{e\left(p^{\alpha}\right)}\right)\right) \cong \mathbf{Z}_{p^{\alpha}}^{e\left(p^{\alpha}\right)}$ is almost identical to the one given for $p^{\alpha} \geqslant 17$. Our upper bound becomes $M_{1}^{(n)}\left(\mathbf{Z}_{p^{\alpha}}^{e\left(p^{\alpha}\right)}\right) \leqslant S_{0}\left(p^{\alpha}, 1,1\right)+S_{0}\left(p^{\alpha}, 2, e\left(p^{\alpha}\right)\right)+1$.

We now pass to the determination of an upper bound for the minimum $M_{0}^{(n)}(A)$ of points in a pure $n$-complex $K$ such that $\Gamma_{0}(K) \cong A$. Again to avoid small exceptional cases we assume that the elementary divisors $p^{\alpha}$ of $A$ satisfy $p^{\alpha} \geqslant 9$. We define the complement $\bar{K}$ of a pure $n$-complex $K$ to be the pure $n$-complex whose point set is $V(K)$ and whose set of maximal simplexes $\bar{K}_{(n)}$ is given by $\bar{K}_{(n)}=\{\alpha \subset$ $\left.V(K):|\alpha|=n+1, \alpha \notin K_{(n)}\right\}$.

THEOREM 10. Let $A$ be a finite abelian group having canonical factorization $A=\Pi \mathbf{Z}_{p^{\alpha}}^{e\left(p^{\alpha}\right)}$. Suppose that $p^{\alpha} \geqslant 9$ for all elementary divisors $p^{\alpha}$ of $A$. Then the minimum number of points $M_{0}^{(n)}(A)$ in an $n$-complex $K$ satisfying $\Gamma_{0}(K) \cong A$ satisfies

$$
M_{0}^{(n)}(A) \leqslant n-2+\sum_{p^{\alpha} \mid A} p^{\alpha} e\left(p^{\alpha}\right)
$$

Proof. We proceed by constructing a complex having point group $\Gamma_{0}$ isomorphic to $\mathbf{Z}_{p^{\alpha}}^{e\left(p^{\alpha}\right)}$ for each elementary divisor $p^{\alpha}$ of $A$, and then we combine these in a certain way to get a complex with point group $A$. This last complex will yield the claimed upper bound.

Let us begin with dimension $n=2$. For any $p^{\alpha}$ dividing $A$, Theorem 4 implies that there exists $r$ such that $\Gamma_{0}\left(C\left(p^{\alpha}, r\right)\right) \cong \Gamma_{2}\left(C\left(p^{\alpha}, r\right)\right) \cong \mathbf{Z}_{p^{\alpha}}$. We let $S_{1}\left(p^{\alpha}\right)=$ $C\left(p^{\alpha}, r\right)$, and we define $S_{k}\left(p^{\alpha}\right)$ inductively for $k \geqslant 2$ by $S_{k}\left(p^{\alpha}\right)=\bar{S}_{k-1} \cup C\left(p^{\alpha}, r\right)$. A simple induction shows that $\Gamma_{0}\left(S_{k}\left(p^{\alpha}\right)\right)=\mathbf{Z}_{p^{\alpha}}^{k}$, and $\left|S_{k}\left(p^{\alpha}\right)_{(0)}\right|=k p^{\alpha}$. 
We may now form the complexes with group $A$ by combining disjoint unions of, and cones over the complexes $S_{k}\left(p^{\alpha}\right)$ defined above. Define $K_{0}^{(2)}(A)$ by $K_{0}^{(2)}(A)=$ $\cup_{p^{\alpha} \mid A} S_{e\left(p^{\alpha}\right)}\left(p^{\alpha}\right)$, and observe that $\Gamma_{0}\left(K_{0}^{(2)}(A)\right) \cong A$. Now for each $n \geqslant 3$ let $K_{0}^{(n)}(A)$ $=C_{n}\left(K_{0}^{(2)}(A)\right)$, and get $\Gamma_{0}\left(K_{0}^{(n)}(A)\right) \cong A$. We therefore obtain the desired bound

$$
M_{0}^{(n)}(A) \leqslant\left|K_{0}^{(n)}(A)_{(0)}\right|=n-2+\sum_{p^{\alpha} \mid A} p^{\alpha} e\left(p^{\alpha}\right),
$$

and the theorem is proved.

We may now discuss cases where the bounds for $M_{1}^{(n)}(A)$ and $M_{0}^{(n)}(A)$ derived in Theorems 8, 9, and 10 are in fact the exact values of $M_{1}^{(n)}(A)$ and $M_{0}^{(n)}(A)$. Toward this end, we need the following lemma.

As a convention, we say that a group $G$ acts faithfully on a set $X$ if and only if there exists an injection $G \rightarrow \Sigma_{|X|}$ of $G$ into the symmetric group on $|X|$ letters.

LEMMA 11.1. Suppose an abelian group $A=\prod \mathbf{Z}_{p^{\alpha}}^{e\left(p^{\alpha}\right)}$ acts faithfully on a set $X$. Then $|X| \geqslant \sum_{p^{\alpha} \mid A} p^{\alpha} e\left(p^{\alpha}\right)$.

Proof. Suppose that $A$ acts faithfully on $X$ and $|X|$ is a minimum among the cardinalities of all sets on which $A$ acts faithfully.

Let $\Omega_{1}, \Omega_{2}, \ldots, \Omega_{r}$ be the orbits of $A$ in $X$, and let $A_{i}$ be the subgroup of $A$ which fixes some point of $\Omega_{i}$ and hence all of $\Omega_{i}$. Observe that $\left|\Omega_{i}\right|=\left|A / A_{i}\right|$ and hence $|X|=\sum_{i=1}^{r}\left|A / A_{i}\right|$. In fact we may identify the objects of $\Omega_{i}$ in a one-to-one manner with the elements of the group $A / A_{i}$. We also have $\cap_{i=1}^{r} A_{i}=1$ by the faithfulness of the action. Our problem may then be stated as that of minimizing the sum $S=\sum\left|A / A_{i}\right|$ over all collections $\left\{A_{i}\right\}$ of subgroups in $A$ satisfying $\cap_{i} A_{i}=1$.

We claim that $A / A_{i}$ is cyclic of prime power order for all $i$. For if not, then there exists $t$ such that $A / A_{t}$ decomposes into $A / A_{t}=B / A_{t} \times C / A_{t}$, with $B \subset A$ and $C \subset A$ properly. We may then define a new set $X^{\prime}$ by $X^{\prime}=\cup_{i \neq t} \Omega_{i} \cup\{A / B\} \cup$ $\{A / C\}$, and we observe that $A$ acts faithfully on $X^{\prime}$ (by multiplication) with the $r+1$ orbits $\left\{\Omega_{i}\right\}_{i \neq t}, A / B, A / C$. The collection of stabilizer subgroups corresponding to $X^{\prime}$ is $\left\{A_{i}\right\}_{i \neq t}, B, C$ and since $(B \cap C) \subset A_{t}$ this collection has trivial intersection. Now clearly $1 /|B|+1 /|C| \leqslant 1 /\left|A_{t}\right|$ and if equality holds we get $A=\mathbf{Z}_{2} \times \mathbf{Z}_{2}$ in which case the lemma obviously holds. Hence we may assume that $1 /|B|+1 /|C|<1 /\left|A_{t}\right|$, and it follows that

$$
\left|X^{\prime}\right|=\sum_{i \neq t}\left|A / A_{i}\right|+|A / B|+|A / C|<\sum_{i=1}^{r}\left|A / A_{i}\right|=|X|,
$$

contradicting the minimality of $|X|$. It follows that $A / A_{i}$ is prime power cyclic for all $i$ as asserted.

Next we claim that $A<\prod_{i=1}^{r} A / A_{i}$. To see this, define the homomorphism $f$ : $A \rightarrow \prod_{i=1}^{r} A / A_{i}$ by $f(\sigma)=\left(\sigma_{1}, \sigma_{2}, \ldots, \sigma_{r}\right)$ where $\sigma_{i}$ is the image of $\sigma$ in the factor group $A / A_{i}, 1 \leqslant i \leqslant r$. Clearly $f$ is injective since $\cap_{i=1}^{r} A_{i}=1$, and hence $A<$ $\prod_{i=1}^{r} A / A_{i}$ as asserted. 
The proof may now be completed. By the above we have

$$
\prod \mathbf{Z}_{p^{\alpha}}^{e\left(p^{\alpha}\right)}=A<\prod_{i=1}^{r} A / A_{i}=\prod \mathbf{Z}_{q^{\beta}}^{e\left(q^{\beta}\right)},
$$

where the $q^{\beta}$ are prime powers, and $|X|=\sum_{i=1}^{r}\left|A / A_{i}\right|=\sum q^{\beta} e\left(q^{\beta}\right)$. Elementary divisor theory now shows that there is an ordering $\left\{p_{1}^{\alpha_{1}}, p_{2}^{\alpha_{2}}, \ldots, p_{m}^{\alpha_{m}}\right\}$, $\left\{q_{1}^{\beta_{1}}, q_{2}^{\beta_{2}}, \ldots, q_{k}^{\beta_{k}}\right\}, m \leqslant k$, of the $p^{\alpha}$ and $q^{\beta}$ appearing in $A$ and $\Pi A / A_{i}$ respectively such that $p_{i}^{\alpha} \mid q_{i}^{\beta}$ for $1 \leqslant i \leqslant m$. It follows that $|X|=\sum q^{\beta} e\left(q^{\beta}\right) \geqslant \sum p^{\alpha} e\left(p^{\alpha}\right)$, as required.

We may now proceed to apply Lemma 11.1 in proving our exact results.

THEOREM 11. Suppose $A=\prod \mathbf{Z}_{p^{\alpha}}^{e\left(p^{\alpha}\right)}$ is an abelian group. For a fixed prime power $p^{\alpha}$ let $\gamma=I\left(p^{\alpha}\right)$ (determined in Theorem 4) be the number of isomorphism classes among the $C\left(p^{\alpha}, j\right)$ satisfying $\Gamma_{2}\left(C\left(p^{\alpha}, j\right)\right) \cong \mathbf{Z}_{p^{\alpha}}$. Then exact values of $M_{1}^{(n)}(A)$ and $M_{0}^{(2)}(A)$ are given by:

(1) $M_{1}^{(n)}(A)=\sum_{p^{\alpha} \mid A} p^{\alpha} e\left(p^{\alpha}\right)$ if

(a) $n=2$, and $p^{\alpha} \geqslant 9, e\left(p^{\alpha}\right) \leqslant \gamma$ for all $p^{\alpha}$ dividing $A$,

(b) $n=3$, and $p^{\alpha} \geqslant 9, e\left(p^{\alpha}\right) \leqslant \gamma 2^{\gamma-1}$ for all $p^{\alpha}$ dividing $A$,

(c) $n \geqslant 4$, and $p^{\alpha} \geqslant 17$ for all $p^{\alpha}$ dividing $A$.

(2) $M_{0}^{(2)}(A)=\Sigma_{p^{\alpha} \mid A} p^{\alpha} e\left(p^{\alpha}\right)$ if $p^{\alpha} \geqslant 9$ for all $p^{\alpha}$ dividing $A$.

Proof. The upper bound for case (1)(a) follows from Theorem 8, items (1) and (2), and from constructions similar to the ones used in the sketch of the proof but omitted for brevity. The same bounds for cases (1)(b) and (1)(c) follow from Theorem 9, items (4), (5) and (1) respectively. In case (2) the bound $M_{0}^{(2)}(A) \leqslant$ $\sum p^{\alpha} e\left(p^{\alpha}\right)$ follows from Theorem 10 .

The corresponding lower bounds $M_{1}^{(n)}(A) \geqslant \sum_{p^{\alpha} \mid A} p^{\alpha} e\left(p^{\alpha}\right)$ and $M_{0}^{(2)}(A) \geqslant$ $\Sigma_{p^{\alpha} \mid A} p^{\alpha} e\left(p^{\alpha}\right)$ in cases (1)(a)-(1)(c) and (2) follow from Lemma 11.1 which states that the number of objects being acted on, be they points (when $A=\Gamma_{0}(K)$ ) or simplexes (when $A=\Gamma_{n}(K)$ ), must be at least $\Sigma_{p^{\alpha} \mid A} p^{\alpha} e\left(p^{\alpha}\right)$.

Acknowledgement. I would like to thank Professors F. Harary, A. Blass, T. Storer, and L. Babai for useful discussions and encouragement during the course of this research.

I would also like to thank Jeanne McKinney for her typing, and Ruth Engel for making the drawings.

\section{REFERENCES}

1. W. Arlinghaus, The smallest graphs with given abelian group, Thesis, Wayne State University, 1979.

2. L. Babai, On the minimum order of graphs with given group, Canad. Math. Bull. 17 (1974), 467-470.

3. A. K. Dewdney, Extensions and generalizations of graph theorems to complexes and hypergraphs, Thesis, University of Waterloo, 1974.

4. F. Harary, Graph theory, Addison-Wesley, Reading, Mass., 1969.

5. F. Harary and R. Duke, Generalized Ramsey theory. VI: Ramsey numbers for small plexes, J. Austral. Math. Soc. Ser. A 22 (1976), 400-410.

6. F. Harary and E. Palmer, The smallest graph whose group is cyclic, Czechoslovak. Math. J. 16 (1966), $70-71$. 
7. On the point-group and line-group of a graph, Acta Math. Acad. Sci. Hungar. 19 (1968), $263-269$.

8. D. McCarthy and L. Quintas, A stability theory for minimum edge graphs with given abstract automorphism group, Trans. Amer. Math. Soc. 208 (1976), 27-39.

9. The construction of minimal-line graphs with given automorphism group, Topics in Graph Theory (F. Harary, ed.) Annals N. Y. Acad. Sci., New York, 1979.

10. R. L. Meriwether, Smallest graphs with a given cyclic group (unpublished).

11. Z. Miller, Minimum simplicial complexes with given abelian automorphism group, Thesis, University of Michigan, 1979.

12. G. Sabidussi, On the minimum order of graphs with given automorphism group, Monatsh. Math. 63 (1959), 124-127.

13. W. R. Scott, Group theory, Prentice-Hall, Englewood Cliffs, N. J., 1964.

14. I. Vinogradov, Elements of number theory, Dover, New York, 1954.

Department of Mathematics and Statistics, Miami University, Oxford, OHio 45056 